\title{
Modeling of dynamic and post-dynamic recrystallization by coupling a full field approach to phenomenological laws
}

\author{
Ludovic Maire ${ }^{\mathrm{a}, *}$, Benjamin Scholtes ${ }^{\mathrm{a}}$, Charbel Moussa ${ }^{\mathrm{a}}$, Nathalie Bozzolo ${ }^{\mathrm{a}}$, Daniel \\ Pino Muñoz ${ }^{\mathrm{a}}$, Amico Settefrati ${ }^{\mathrm{b}}$, Marc Bernacki ${ }^{\mathrm{a}}$ \\ ${ }^{a}$ MINES ParisTech, PSL - Research University, CEMEF - Centre de mise en forme des matriaux, CNRS \\ UMR 7635, CS 10207 rue Claude Daunesse 06904 Sophia Antipolis Cedex, France. \\ ${ }^{b}$ Transvalor S.A., 694 avenue Maurice Donat, 06250 Mougins, France
}

\begin{abstract}
This paper describes a level set framework for the full field modeling of dynamic and post-dynamic recrystallization in a $3 \mathrm{D}$ polycrystalline material with an accurate description of grains topology at large deformation and application to 304L austenitic stainless steel. Topological evolutions are simulated based on a kinetic law linking the velocity of the boundaries to the thermodynamic driving forces. Recrystallization is modeled by coupling a level set approach to phenomenological laws describing strain hardening mechanism and nucleation criteria. Although the proposed formalism does not consider crystal plasticity because of its computational costs, it enables to reach outstanding dynamic recrystallization computations in a front-capturing finite element framework comparatively to the state of art.

Keywords: microstructures, grain boundaries, recrystallization, metallic materials, finite elements, level set.
\end{abstract}

Funding: This research did not receive any specific grant from funding agencies in the public, commercial, or not-for-profit sectors.

\footnotetext{
${ }^{*}$ Corresponding author

Email address: ludovic.maire@mines-paristech.fr (Ludovic Maire)
} 


\section{Introduction}

Hot deformation of metals is responsible of a wide range of phenomena occurring in the microstructure. At high temperatures, the dislocation density is the result of a competition between the increase of the amount of dislocations due to plastic work and 5 its decrease due to dynamic recovery. In general, the increase is faster than the decrease and the dislocation density globally goes up. Once a critical dislocation density is locally reached, the recrystallization mechanism (i.e. nucleation of recrystallized grains) occurs in the microstructure. These recrystallized grains have a low energy compared to the deformed ones. Because of stored energy gradients between recrystallized and non-recrystallized grains, recrystallized grains grow by consuming deformed grains. Simultaneously, dislocation density increases in the recrystallized grains because of plastic deformation. Capillarity effects (leading to the decrease of the total grain boundaries surface) are also present and play a role regarding the evolution of multiple junctions. All these mechanisms acting during hot deformation lead to the so-called discontinuous dynamic recrystallization process (DDRX) [1, 2, 3, 4] which is the classical dynamic recrystallization (DRX) mechanism for low/medium stacking fault energy (SFE) metallic materials such as the 304L austenitic steel considered for application in this work. If the plastic deformation is stopped and the material is still at high temperature, grain boundary migration is still active because of stored energy gradients and capillarity effects. Furthermore, the nucleation mechanism can still occur in some hardened grains. These mechanisms appearing after hot deformation lead to the so-called post-dynamic recrystallization process (PDRX) [5, 6]. A review of dynamic recrystallization phenomena occurring in metals has been recently proposed in [4].

25 Since many decades, phenomenological recrystallization models have been proposed like the well-known Avrami relationship aiming to describe the global recrystallized fraction during hot deformation [7, 8, 9]. Many years later, new recrystallization models emerged with the intention to implicitly describe the microstructure by considering spherical grains [10, 11, 12, 13, 14]. In these models, each physical mechanism is described by a physical or phenomenological law. Grains evolve by computing a velocity 
between each grain and the averaged microstructure: these kinds of models are often called mean field models. The main advantage of these models is related to the fact that they provide good results in terms of recrystallized fraction or mean grain size and they also provide additional information in terms of grain size distribution and dislocation density distribution. However, the topology of grains and the interactions between neighboring grains cannot be described using mean field models. Thus several assumptions on the grain mean curvature or on nucleation sites can considerably limit their use.

In parallel, finer approaches called full field models have emerged in the last decades. crystal scale, which makes the modeling of local phenomena such as abnormal grain growth possible. A review of the most significant numerical methods is given in [15]. Probabilistic voxel-based approaches such as Monte Carlo [16, 17] and cellular automata [18, 19] are very popular. These models consider uniform grids composed of cells to model microstructure and stochastic laws to predict the motion of interfaces. These simulations are efficient in term of computational cost and the scalability is excellent. On the other hand, deterministic approaches, based on the resolution of partial differential equations, are more accurate in the description of the involved physical mechanisms although they are numerically more expensive. For instance, the vertex 50 approach [20] is based on an explicit description of interfaces in terms of vertices. Interfaces motion is imposed at each increment by computing the velocity of a set of points. A major drawback of this approach is related to the complexity of handling all the possible topological events, such as disappearance and appearance of new grains, which is not straightforward especially in 3D. Other deterministic approaches avoid these topological problems since they are based on an implicit description of the interfaces: the phase-field [21, 22] and the level set (LS) method [23, 24]. The major limitation of these two methods is generally the computational cost but recent improvements of this weakness should be emphasized [22, 25]. LS simulations in context of regular grids and Fourier transform resolution can be found for grain growth [26, 27] and for static recrystallization [25] modeling. When global or local meshing/remeshing operations have to be considered (large deformation, accuracy in the grain interfaces, 
presence of second phase particles...), LS approach in context of unstructured finite element (FE) mesh has to be considered [24, 28, 29, 30, 31, 32]. The numerical LS framework developed in [32] is considered and enhanced for DRX modeling in the following.

Several DRX models based on a full field approach exist in the literature. In the last decades, [33] has already proposed a 2D model of DRX based on the cellular automata method for interfaces tracking and phenomenological laws to describe hardening and nucleation mechanisms. More recently in [30], a 2D model considering a FE based LS approach is proposed to model DRX. In [34], a coupled crystal plasticity and cellular automata approach has been developed to model DRX in a titanium alloy. In [35], a combined crystal plasticity and vertex model is proposed in 2D to model DRX for large deformation. In [36], a coupled crystal plasticity and cellular automata approach 75 is used in 2D to model DRX in magnesium alloys. In [37], a 3D model based on the phase-field method coupled with a fast Fourier transform based elasto-viscoplastic model is proposed to model DRX in copper. In [38], a 2D cellular automata model has been used to model DRX in Inconel 718 alloy. In [39], a coupled crystal plasticity and cellular automata approach has been proposed in 3D for modeling DRX in titanium alloys. In [40], a 2D cellular automata model coupled to phenomenological laws for strain hardening, recovery and nucleation mechanisms, has been developed to predict microstructural evolutions in a magnesium alloy during hot deformation. In parallel, several analytical models have been recently proposed in order to simulate DRX as a function of the input process conditions [41, 42, 43, 44].

${ }_{85}$ Although literature already provides a large number of papers on full field DRX models, a major drawback is the difficulty to handle grains topology and morphology for large deformation (for instance $\varepsilon>0.2$ ), which is a major limitation since many industrial processes go well further in deformation. Furthermore, crystal plasticity is generally used in full field DRX models since it predicts accurately the local evolution 90 of dislocation density at the polycrystal scale. However, the use of crystal plasticity often leads to prohibitive computational costs. Finally, most of DRX full field models are developed in 2D because of numerical issues and computational costs. Microstructural 
evolutions present a three-dimensional character, therefore results obtained from 2D models may not be representative of a real 3D process. Some limitations of 2D models are related to the location of nuclei, their initial size or growth rates.

In the present work, a 3D-FE model based on the LS method in context of unstructured tetrahedral mesh is proposed to model the DRX and PDRX phenomena with application to $304 \mathrm{~L}$ steel. This model is able to describe grain topology at large deformation (for instance $\varepsilon>2$ ) with relatively low computational costs. The equations proposed and validated in a mean field model [13, 12] are used in this model to simulate the nucleation, work hardening and recovery mechanisms while the LS approach coupled to a remesher provides an accurate tracking of interfaces (i.e. grain boundaries) all along the simulation. The first section of the paper introduces the modeling of the grain boundaries network and grain boundary kinetic. This section is followed by the presentation of the main constitutive equations of the models. Then, a sensitivity study of optimal model parameters (mesh size, initial number of grains, deformation step, time step and critical nucleus radius) on microstructural responses of the model is investigated. The subsequent section presents a comparison between our full field model and a mean field model of the literature [12] in given thermomechanical conditions.

\section{Microstructure and grain boundary migration}

\subsection{Representation of the initial grain boundaries network and energy field}

The model considered in this paper is based on a LS description of the interfaces within a FE framework [24, 29, 30, 15]. First, grain interfaces are virtually generated by using the Voronoï tessellation or Laguerre-Voronoï method [45, 46, 47, 48, 49]. The virtual interfaces are then described into an unstructured FE mesh thanks to LS functions (see appendix A for more details about the generation of the grain boundaries network and appendix B for an introduction of the LS method in the considered polycrystalline framework). 
A 3D digital microstructure representative of 304L steel has been generated according to a Laguerre-Voronoï algorithm and using the LS approach within a FE framework. The grain size follows a log-normal distribution and result is presented in Fig. 11(a). The mean grain radius $\langle R\rangle$ and the standard deviation $\sigma_{\mathrm{R}}$ of the distribution are $60 \mu \mathrm{m}$ and $10 \mu \mathrm{m}$, respectively. The color code corresponds to the equivalent spherical grain radius. The number of grains in the Representative Volume Element (RVE) is around 8000 , the number of mesh elements is around 3.5M and $22 \mathrm{LS}$ functions are used.

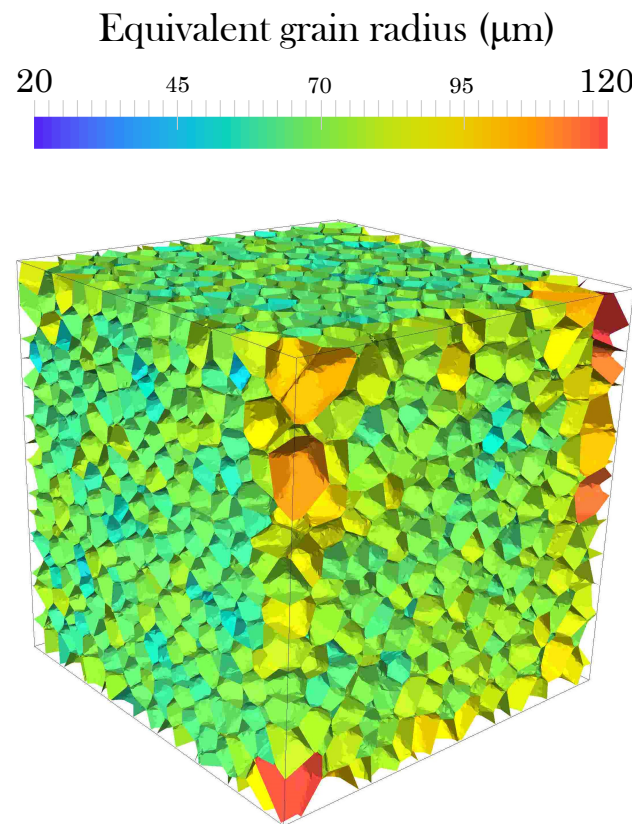

(a)

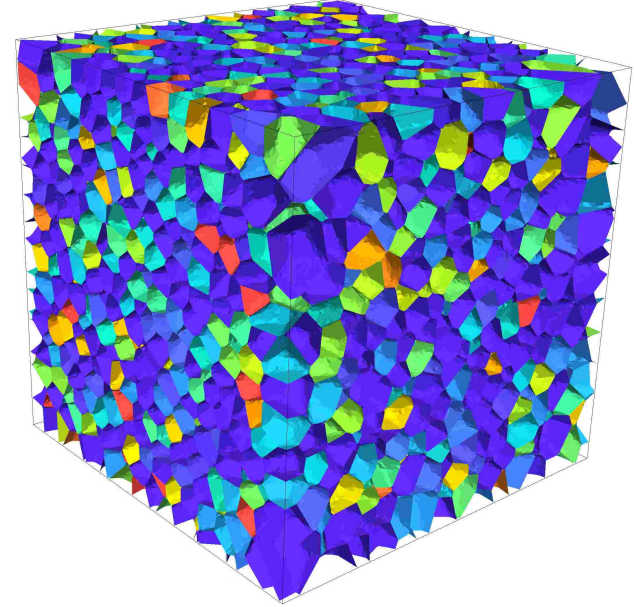

(b)

Figure 1: 3D digital microstructure generated according to a Laguerrre-Voronoï algorithm following a Lognormal distribution $\left(\langle R\rangle=60 \mu \mathrm{m} ; \sigma_{\mathrm{R}}=10 \mu \mathrm{m}\right)$. (a) Color code corresponds to the equivalent spherical grain radius. (b) Color code corresponds to the energy field considered constant per grain and generated from a Gaussian distribution $\left(\langle E\rangle=1 \mathrm{e}-5 \mathrm{~J} \cdot \mathrm{mm}^{-3} ; \sigma_{\mathrm{E}}=2 \mathrm{e}-4 \mathrm{~J} \cdot \mathrm{mm}^{-3}\right)$.

At the initial stage of the simulation, an energy field considered constant per grain is defined in the microstructure. This energy field can be generated either according to a particular distribution (Gaussian, log-normal, bimodal) or from experimental data. In the case presented in Fig. 1 (b) representative of 304L steel, an initial energy field 
is generated from a Gaussian distribution. The mean energy $\langle E\rangle$ and the standard deviation $\sigma_{\mathrm{E}}$ of the distribution are 1e-5 J.mm ${ }^{-3}$ and $2 \mathrm{e}-4 \mathrm{~J} . \mathrm{mm}^{-3}$, respectively. An energy field $E$ is related to a dislocation density field $\rho$ and vice versa according to the following relation :

$$
E=\tau \rho,
$$

where $\tau$ represents the dislocation line energy and is considered as material dependant.

\subsection{Grain boundary migration}

As detailed in the introduction, during a process at high temperature, grain interfaces migrate due to the stored energy gradient across the interface and due to the grain boundary mean curvature which is the sum of the main curvatures in the $3 \mathrm{D}$ case. Grain boundary migration is modelled in the considered model by using the strategy adopted in [32]. Since we used the formalism of equations proposed and validated in [13, 12], we also made, although this consideration has not at yet a clear physical sense, the velocity term dependent on the strain rate by considering an additional term $\delta(\dot{\varepsilon})$ into the velocity formula (see appendix $\mathrm{C}$ for more details concerning the modeling of the grain boundary migration kinetic and about the term $\delta(\dot{\varepsilon})$ ).

\section{Dynamic and post-dynamic recrystallization modeling}

\subsection{Strain hardening and recovery mechanisms}

During plastic deformation, dislocations density increases in the microstructure due to strain hardening, resulting in an increase of the stored energy. However, a part of dislocations can also annihilate due to dynamic recovery. The strain hardening and recovery mechanisms appearing during hot deformation, can be modeled at different scales: at a local scale with crystal plasticity [35, 36, 50] or at a macroscopic scale with phenomenological laws [51, 52, 53]. In this model, phenomenological laws are considered to limit the computational cost of the 3D simulations, thus none resolution of a mechanical problems is considered. The deformation is simply modeled by applying a normal velocity on different faces of the RVE so as to respect a given strain rate 
and a velocity gradient. Each mesh node is thus displaced according to these velocities. During deformation, the faces of the RVE remain plane (homogeneous material assumption) and the volume conservation of the RVE is ensured (incompressible material).

Considering $N_{\mathrm{G}}$ grains in the microstructure, the averaged dislocation density field obtained from Eq. 1 in each grain $j$ noted $\left\langle\rho_{\mathrm{j}}\right\rangle$, is assumed to evolve according to the Yoshie-Laasraoui-Jonas law [53] :

$$
\frac{\partial\left\langle\rho_{\mathrm{j}}\right\rangle}{\partial \varepsilon_{\mathrm{eff}}^{\mathrm{p}}}=K_{1}-K_{2}\left\langle\rho_{\mathrm{j}}\right\rangle
$$

where $\varepsilon_{\text {eff }}^{\mathrm{p}}$ denotes the effective plastic strain, $K_{1}$ and $K_{2}$ are two constants which represent the strain hardening and dynamic recovery term, respectively. At each time increment, this differential equation is solved with an Euler explicit method, i.e. :

$$
\frac{\left\langle\rho_{\mathrm{j}}\right\rangle^{(\mathrm{t}+\Delta \mathrm{t})}-\left\langle\rho_{\mathrm{j}}\right\rangle^{\mathrm{t}}}{\Delta \varepsilon}=K_{1}-K_{2}\left\langle\rho_{\mathrm{j}}\right\rangle^{\mathrm{t}}
$$

where $\Delta \varepsilon$ is equal to $\dot{\varepsilon}_{\text {eff }}^{\mathrm{p}} \times \Delta t$ with $\Delta t$ the time step, which leads to the final equation :

$$
\left\langle\rho_{\mathrm{j}}\right\rangle^{(\mathrm{t}+\Delta \mathrm{t})}=K_{1} \Delta \varepsilon+\left(1-K_{2} \Delta \varepsilon\right)\left\langle\rho_{\mathrm{j}}\right\rangle^{\mathrm{t}}
$$

When a grain boundary migrates, the swept area is almost free of defects, i.e. dislocationsfree. This phenomenon is traduced by a decrease of the dislocation density in growing grains. Thus a minimal dislocation density $\rho_{0}$, which is material dependant, is attributed to swept areas. Then the new dislocation density is averaged in each grain following the equation :

$$
\left\langle\rho_{\mathrm{j}}\right\rangle^{(\mathrm{t}+\Delta \mathrm{t})} V^{(\mathrm{t}+\Delta \mathrm{t})}=\left\langle\rho_{\mathrm{j}}\right\rangle^{\mathrm{t}} V^{\mathrm{t}}+\Delta V \rho_{0},
$$

where $\Delta V$ represents the swept volume between the instants $t$ and $(t+\Delta t)$. Fig. 2 shows the evolution of a dislocation density field in a grain between $t$ and $(t+\Delta t)$ due to strain hardening, dynamic recovery and grain boundary migration mechanisms. 


\section{- Grain boundary at $t$ --- Grain boundary at $t+\Delta t$}

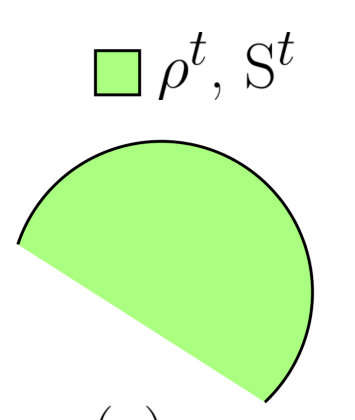

(a)

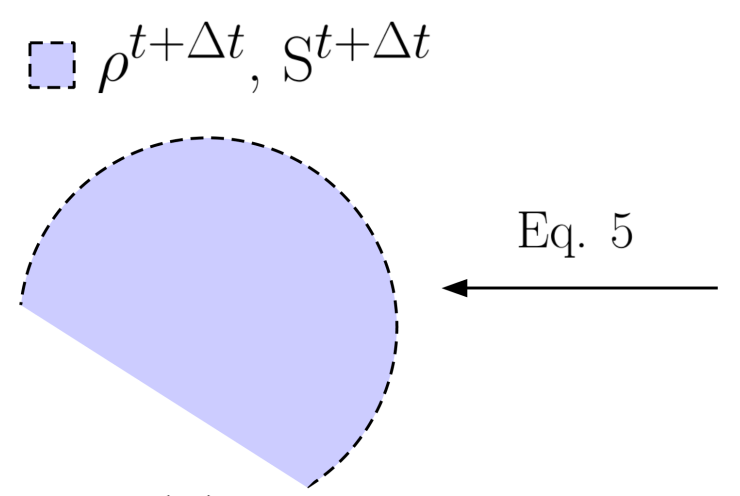

(d)

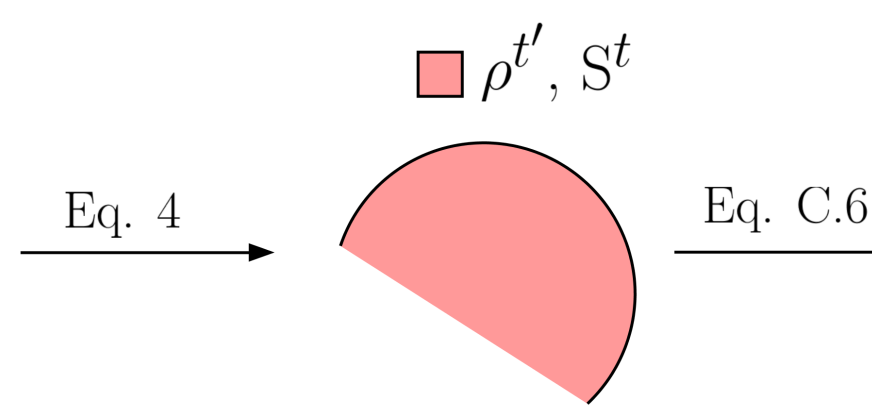

(b)

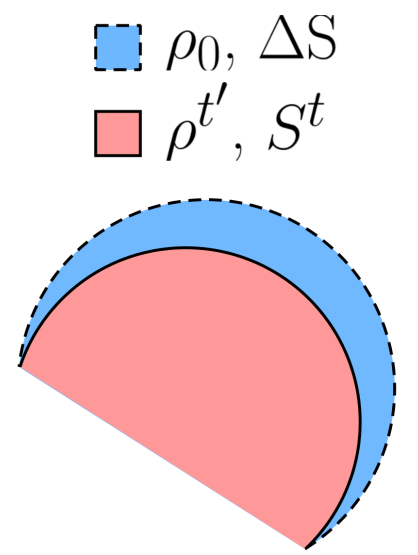

(c)

Figure 2: $2 \mathrm{D}$ scheme illustrating the evolution of a dislocation density field of a single grain due to the mechanisms of strain hardening and dynamic recovery(from (a) to (b)), grain boundary migration(from (b) to (c)) and decrease of dislocation density due to boundary migration(from (c) to (d)) between the instants $t$ and $(t+\Delta t)$ of the simulation. 
The PDRX mechanism is taken into account after deformation by modeling the migration of grain boundaries (see section 2.2.), the decrease of dislocation density due to boundary migration given by Eq. 5 and the static recovery due to annihilation of dislocations given by Eq. 6:

$$
\left\langle\dot{\rho_{\mathrm{j}}}\right\rangle=-K_{\mathrm{s}}\left\langle\rho_{\mathrm{j}}\right\rangle,
$$

where $K_{\mathrm{S}}$ is a temperature dependant parameter which represents the static recovery term and a superposed dot denotes differentiation with respect to time. During PDRX, no nucleation of recrystallized grains is considered, thus the following section on nucleation only concerns DRX mechanism.

The mean flow stress $\sigma_{\mathrm{j}}$ in the $j^{\text {th }}$ grain is computed during deformation from its average dislocation density $\left\langle\rho_{\mathrm{j}}\right\rangle$ using the Taylor equation :

$$
\sigma_{\mathrm{j}}=\sigma_{0}+\alpha \mu b \sqrt{\left\langle\rho_{\mathrm{j}}\right\rangle},
$$

where $\sigma_{0}$ is a dislocations-free yield stress and $\alpha$ is a constant. Then the total flow stress $\langle\sigma\rangle$ is calculated as a volume average of the flow stresses in all grains :

$$
\langle\sigma\rangle=\frac{\sum \sigma_{\mathrm{j}} V_{\mathrm{j}}}{V_{\mathrm{tot}}}
$$

\subsection{Nucleation mechanism}

When enough energy is accumulated in the material due to plastic deformation, some dislocation networks can develop within grains and tend to form new recrystallized grains called nuclei, mainly located at grain boundaries [54]. Different criteria need to be verified locally in order to obtain a substructure that becomes a nucleus: a mobile high-angle grain boundary has to be formed by the nucleation event, a high stored energy gradient across the interface must be present in order to provide enough positive driving pressure for counter the capillarity effects applied on the nucleus. In the considered framework, as $\gamma_{b}$ is assumed isotropic, only the stored energy and the critical nucleus radius is taken into account for nucleation event. 


\subsubsection{Nuclei location}

The adopted strategy concerning the creation of new nuclei in a FE-LS framework is based on the recent developments of Scholtes et al. [32]. In the considered model, we assume that new nuclei of critical radius $\mathrm{r}^{*}$ only appear close to grain boundaries.

This restriction is taken into account by forcing the centers of new nuclei inside a layer $\pm L$ around the interfaces, where $L=r^{*}$ (see Fig. 3 for more details).

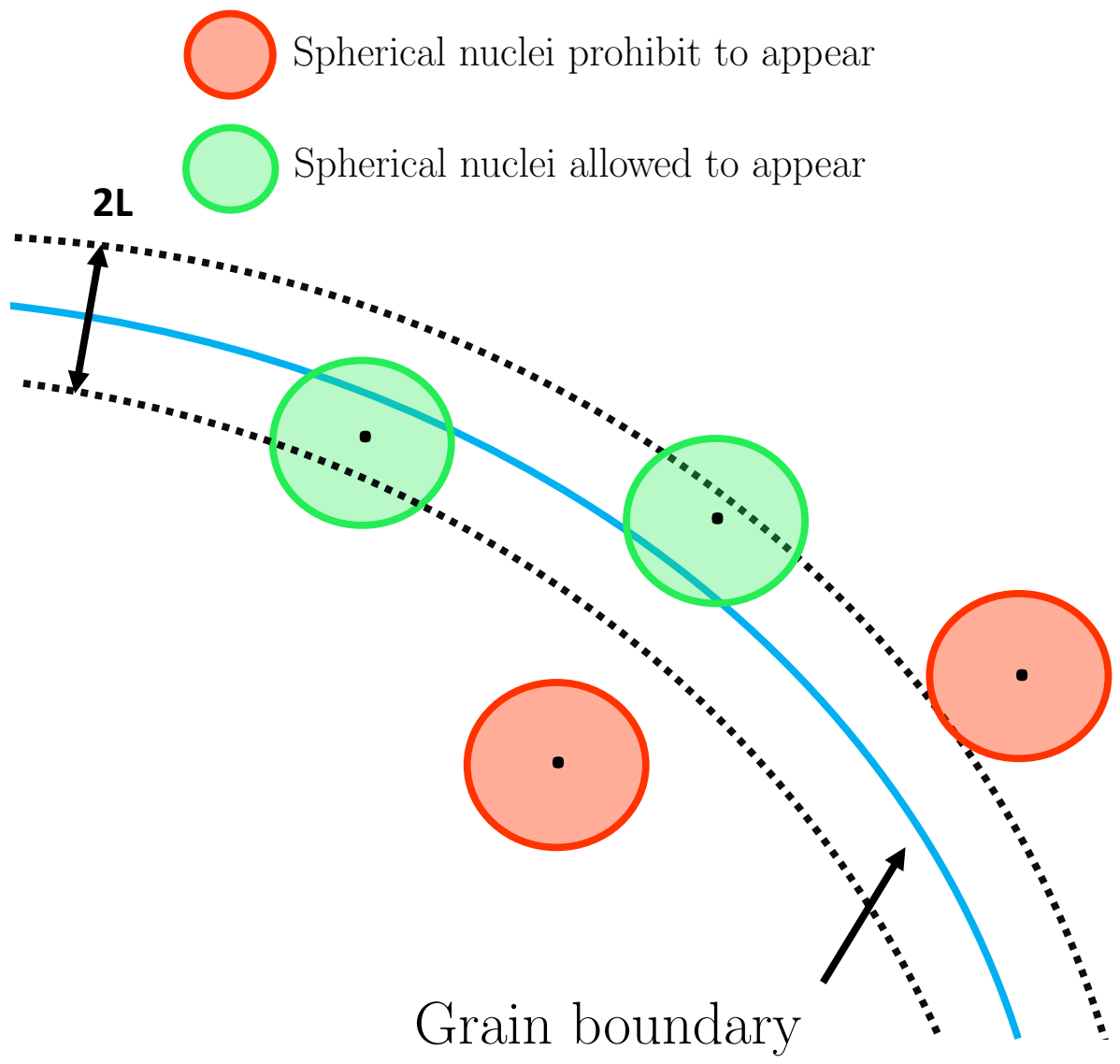

Figure 3: Examples of nuclei that are allowed (green color) and prohibit (red color) to appear depending on the distance from the grain boundary to their centers. 


\subsubsection{Critical stored energy for nucleation}

In the considered model, we assume that a new nucleus can appear if the averaged stored energy in its appearance area reaches a critical value $\rho_{\text {cr }}$ (see Fig. 4). This restriction is taken into account by averaging the energy in the volume occupied by the nucleus before its appearance, and by verifying if this averaged energy reaches $\rho_{\mathrm{cr}}$. (see Fig. 4 for more details). If this condition is verified and if its position satisfies the condition presented previously, the new nucleus can be created with a stored energy $\rho_{0}$.

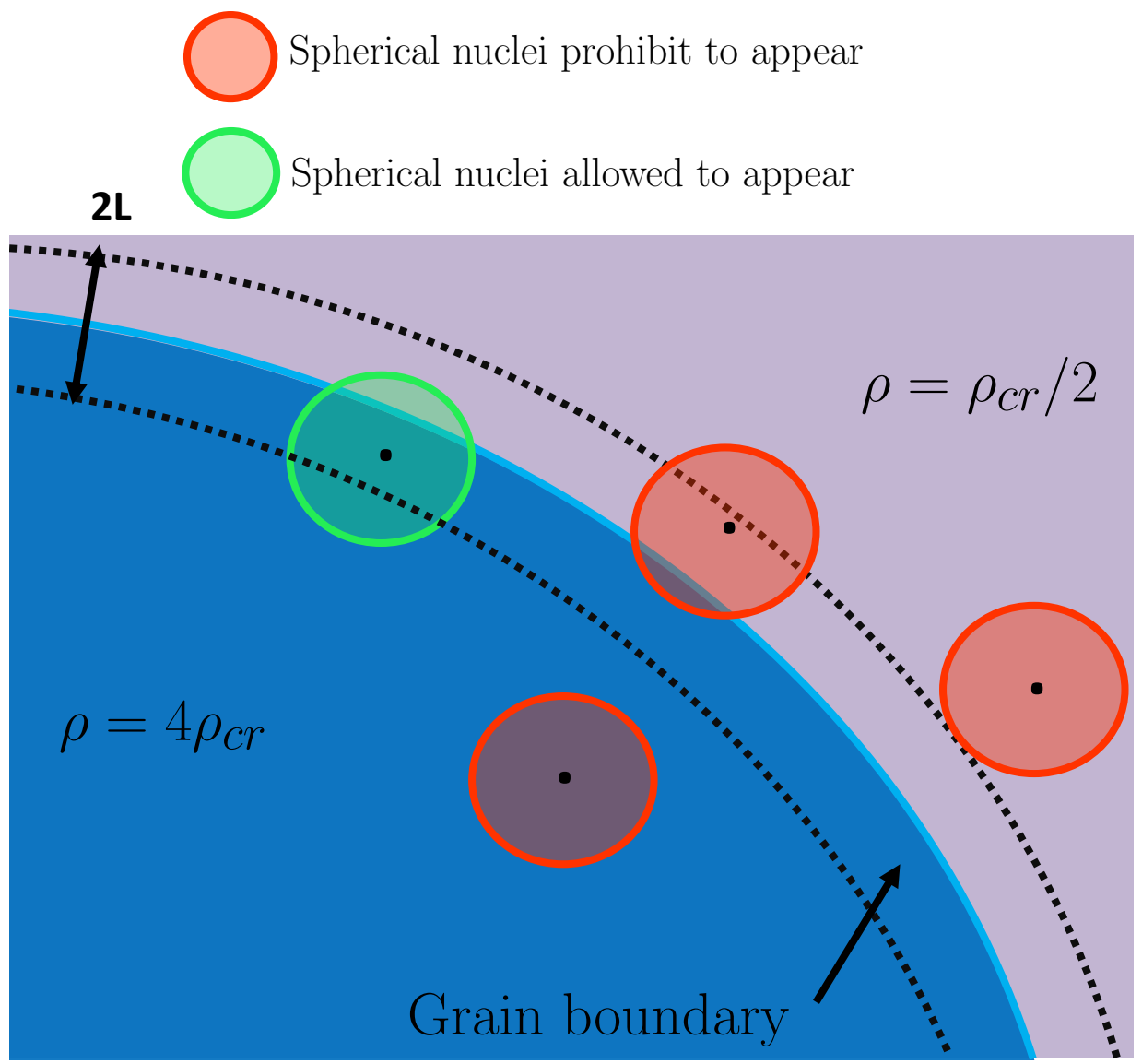

Figure 4: Examples of nuclei allowed (green) and prohibit (red) to appear depending on whether the averaged stored energy in the volume occupied by each nucleus before its appearance reached the critical dislocation density $\rho_{\mathrm{cr}}$. 
Concerning the computing of $\rho_{\mathrm{cr}}$, a first approximation is made and an iterative calculation of $\rho_{\mathrm{cr}}$ is done according to the following equation [13] :

$$
\rho_{\mathrm{cr}}=\left[\frac{-2 \gamma_{\mathrm{b}} \dot{\varepsilon} \frac{K_{2}}{M_{\mathrm{b}} \delta(\dot{\varepsilon}) \tau^{2}}}{\ln \left(1-\frac{K_{2}}{K_{1}} \rho_{\mathrm{cr}}\right)}\right]^{1 / 2}
$$

where $M_{\mathrm{b}}$ is the grain boundary mobility and $\gamma_{\mathrm{b}}$ is the grain boundary energy.

The influence of the temperature on $\rho_{\text {cr }}$ is taken into account in Eq. 9 thanks to the parameters $K_{2}$ and $M_{\mathrm{b}}$ while the influence of the strain rate on $\rho_{\mathrm{cr}}$ is taken into account in Eq. 9 thanks to the parameters $K_{1}, K_{2}, \dot{\varepsilon}$ and $\delta(\dot{\varepsilon})$. It is assumed that $\rho_{\text {cr }}$ increases when decreasing temperature or increasing strain rate.

\subsubsection{Nucleus critical radius}

When a new nucleus appears in the microstructure, its critical radius must be high enough so that its stored energy counters the capillarity forces applied by the neighboring grains. This corresponds to the condition when the stored energy of the material is large enough to overcome the capillarity effects exerted by neighboring grains on the nucleus. This condition is approximated by the so-called Bailey-Hirsch criterion [55] :

$$
r^{*}=\omega \frac{2 \gamma_{\mathrm{b}}}{\rho_{\mathrm{cr}} \tau},
$$

where $\omega>1$ is a safety factor ensuring that the created nucleus has a required driving force for growth. Indeed the Eq. 10 with $\omega=1$ is based on the assumption that a new nucleus is perfectly spherical but in the present model, depending on the mesh size, the nucleus cannot be perfectly spherical, which justifies the use of the safety factor $\omega$. The value of $\omega$ will be investigated in the section 4 . 


\subsubsection{Nucleation rate}

The nucleation rate $\dot{V}$ representing a volume of nuclei per unit of time, is calculated according to a variant of the proportional nucleation model of Peczak and Luton [56] :

$$
\dot{V}=K_{\mathrm{g}} \Phi \Delta \mathrm{t},
$$

where $K_{\mathrm{g}}(\mathrm{T}, \dot{\varepsilon})$ is a probability coefficient related to the thermo-mechanical conditions, i.e. the temperature and the effective plastic strain rate and $\Phi$ represents the total boundary area (in a necklace-type nucleation) or total volume (in a bulk-type nucleation) of grains verifying $\rho_{\mathrm{i}}>\rho_{\mathrm{cr}}$.

For better understanding of the coupling between LS method and recrystallization governing laws, a flow chart summarizing the resolution method of the present model for a given process time is presented in Fig. 5 


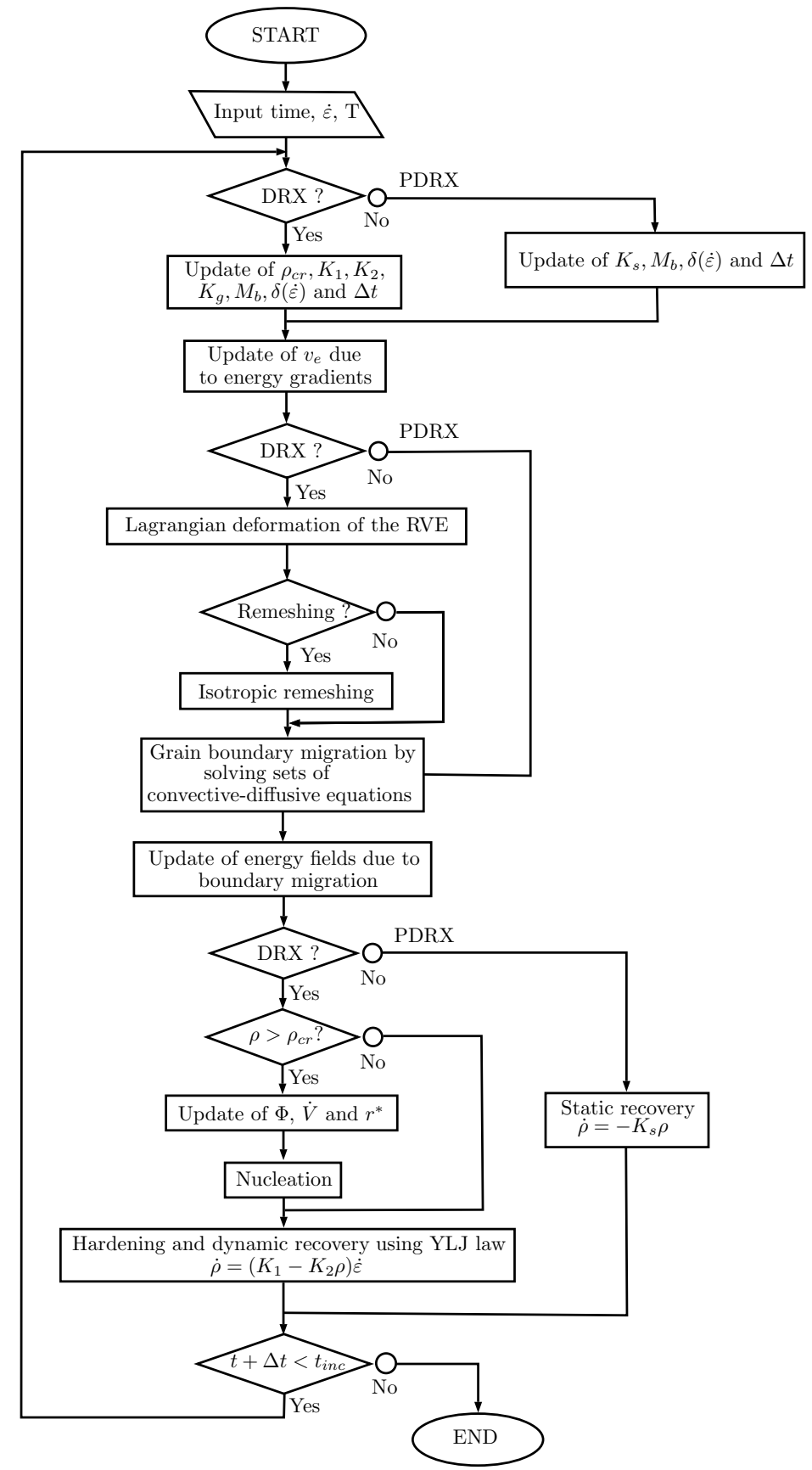

Figure 5: Scheme describing the resolution method of the present model for a given process time, strain rate $\dot{\varepsilon}$ and temperature $\mathrm{T}$. 


\section{Sensitivity study of the present model}

The DRX and PDRX model considered many parameters which have to be correctly initialized in order to model as accurately as possible the microstructural mechanisms, while keeping relatively low computational costs. This section is dedicated to a sensitivity study of initial parameters leading to converged results of DRX (see 4.1.) and then PDRX (see 4.2.).

\subsection{Dynamic recrystallization}

An identification of the optimal mesh size, initial number of grains, deformation step and critical nucleus radius of the DRX model leading to converged results while keeping low computational costs is done in this section.

\section{Mesh size}

A single nucleus is considered and the mesh size is calibrated so as to obtain a good description of this nucleus in terms of surface and volume. The isotropic mesh size must be small enough to correctly describe the nucleus mean curvature but also reasonable in order to limit the computational cost. Thus four spherical nuclei of radius $20 \mu \mathrm{m}$ are generated using four different mesh sizes and results are presented in Fig. 6 The quantity $\xi$ corresponds to the ratio between the nucleus radius $r^{*}$ and the mesh size and is reported below each nucleus in Fig. 6 The quantity $E_{v}$ corresponds to the $\mathrm{L}^{1}$ error between the volume of the generated nucleus of radius r* (presented in Fig. 6) and the volume of a sphere of radius $\mathrm{r}^{*}$. The error between the surfaces is also measured and noted $\mathrm{E}_{\mathrm{s}}$. These values are reported below each nucleus in Fig. 6 


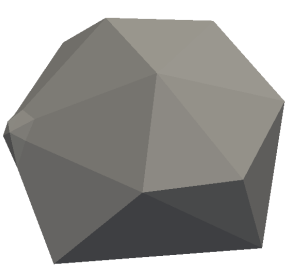

(a) $\xi=1.0, E_{v}=31 \%, E_{s}=27 \%$

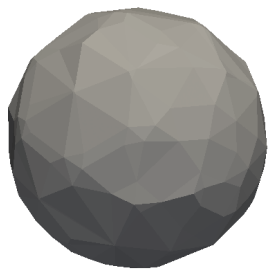

(c) $\xi=2.1, E_{v}=4.3 \%, E_{s}=4,3 \%$

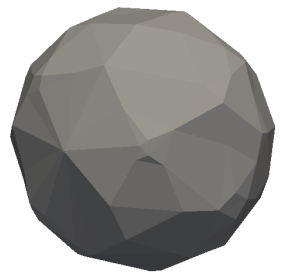

(b) $\xi=1.5, E_{v}=7.7 \%, E_{s}=7.1 \%$

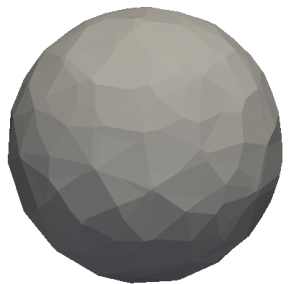

(d) $\xi=2.6, E_{v}=2.8 \%, E_{s}=2.5 \%$

Figure 6: Four nuclei generated according to different mesh sizes. $\xi$ corresponds to the ratio between the nucleus radius $r^{*}$ and the mesh size. $E_{\mathrm{V}}$ (resp. $E_{\mathrm{S}}$ ) corresponds to the $\mathrm{L}^{1}$ error between the volume (resp. surface) of the generated nucleus and the volume (resp. surface) of a sphere of same radius.

It is observed that in each case, the $E_{\mathrm{v}}$ error is close to the $E_{\mathrm{S}}$ error. The nucleus presented in Fig. 6(a) has large $E_{\mathrm{v}}$ and $E_{\mathrm{s}}$ errors (31\% and 27\%, respectively). The three other nuclei presented in Fig. 6(b), (c) and (d) give $E_{\mathrm{v}}$ and $E_{\mathrm{s}}$ errors lower than $10 \%$, leading to a better description of the nuclei in these three cases. A ratio $\xi \simeq 2$ between the nucleus radius and the mesh size is chosen as a good compromise between a minimum number of mesh elements into the nucleus and low $E_{\mathrm{v}}$ and $E_{s}$ errors. Thus, the future number of mesh elements in the simulations will always be chosen so as to respect a ratio $\xi \simeq 2$.

\section{Initial number of grains}

Several full field simulations of DRX have been performed using the considered model for a temperature of $1273^{\circ} \mathrm{K}$ and a strain rate of $0.01 \mathrm{~s}^{-1}$ during $300 \mathrm{~s}$. The deformation of the RVE is idealized at each increment by updating all mesh nodes coordinates at 
the instant $t+\Delta t$ thanks to the relations :

$$
\begin{array}{r}
x^{\mathrm{t}+\Delta \mathrm{t}}=x^{\mathrm{t}}, \\
y^{\mathrm{t}+\Delta \mathrm{t}}=(1+\dot{\varepsilon} \Delta t) y^{\mathrm{t}}, \\
z^{\mathrm{t}+\Delta \mathrm{t}}=(1-\dot{\varepsilon} \Delta t) z^{\mathrm{t}},
\end{array}
$$

with $\left(x^{\mathrm{t}} ; y^{\mathrm{t}} ; z^{\mathrm{t}}\right)$ and $\left(x^{\mathrm{t}+\Delta \mathrm{t}} ; y^{\mathrm{t}+\Delta \mathrm{t}} ; z^{\mathrm{t}+\Delta \mathrm{t}}\right)$ the coordinates of a mesh node at the instants $t$ and $t+\Delta t$ respectively, considering the $(0, \vec{x}, \vec{y}, \vec{z})$ Cartesian coordinate system (see Fig. 7), $\dot{\varepsilon}$ the strain rate following the direction of solicitation (i.e. direction $\vec{z}$ ) and $\Delta t$ the time increment. These equations lead to an idealized channel-die case, where the six faces remain plane during the simulated process. Computations were performed on three nodes of $24 \mathrm{CPU}$ processors each in order to compare computational costs. The variation of the initial number of grains is done by keeping the same initial mean grain radius $\left(\simeq 60 \mu \mathrm{m}\right.$ ) and by varying the RVE size (from $0.14 \mathrm{~mm}^{3}$ to $0.34 \mathrm{~mm}^{3}$ ). The safety factor $\omega$ (Eq. 10) is taken equal to 2, leading to a critical nucleus radius of $7 \mu \mathrm{m}$. The mesh size is fixed to $3 \mu \mathrm{m}$ in order to validate the ratio $\xi \simeq 2$. The step deformation is taken low enough (2\%) to ensure a good convergence of calculations. Microstructures are presented in Fig. 7 and results in terms of recrystallized fraction, mean grain radius, mean dislocation density, computational cost, grain radius distribution and dislocation density distribution are plotted in Fig. 8. The mean grain radius $\left\langle R_{\mathrm{vol}}\right\rangle$ and the mean dislocation density $\left\langle\rho_{\mathrm{vol}}\right\rangle$ are weighted by grain volume. The distributions are plotted for a true deformation $\varepsilon=1$. Only grains from 0 to $12 \mu \mathrm{m}$ have been considered in Fig. 8 e) since these grains represent more than $99 \%$ of the total number of grains in the microstructure. It is clearly observed that a total of 8 grains in the initial microstructure leads to results as close as with a number of 20 or 40 grains (see Fig. 8). Indeed our nucleation model considers a random nucleation on high energy grain boundaries. So during the DRX mechanism when all grains have a sufficient energy, nuclei will appear everywhere at every interfaces and the total number of grains rapidly increases in the microstructure, reducing the influence of the initial number of grains in this case. Two simulations with two different initial number of grains (8 and 27 grains) and exactly the same initial grain radius distribution and energy distribution are presented in Fig. 7 . 
It is clearly observed for a deformation $\varepsilon=1.5$ that the volumic fractions of nuclei are similar in the two cases. Thus it is assumed that the accuracy of the results is sufficient with an initial number of 8 grains. 


\section{Energy $\left(\mathrm{J}_{\mathrm{mm}}^{-3}\right)$}
$1 e^{-7}$
$1.1 \mathrm{e}^{-4}$
$2.2 \mathrm{e}^{-4}$
$3.3 \mathrm{e}^{-4}$
$4.4 \mathrm{e}^{-4}$

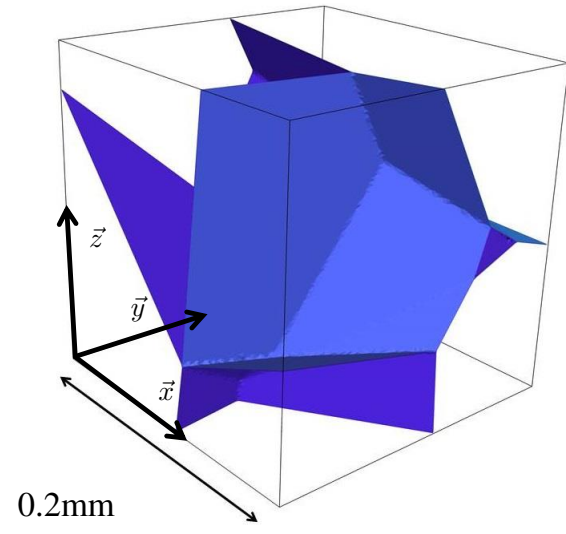

(a) $\varepsilon=0$

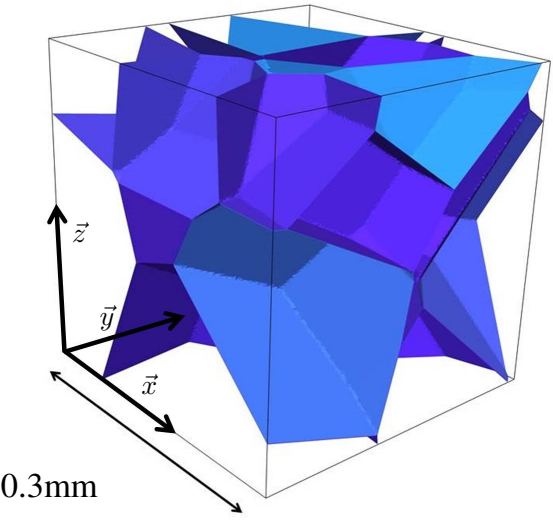

(c) $\varepsilon=0$

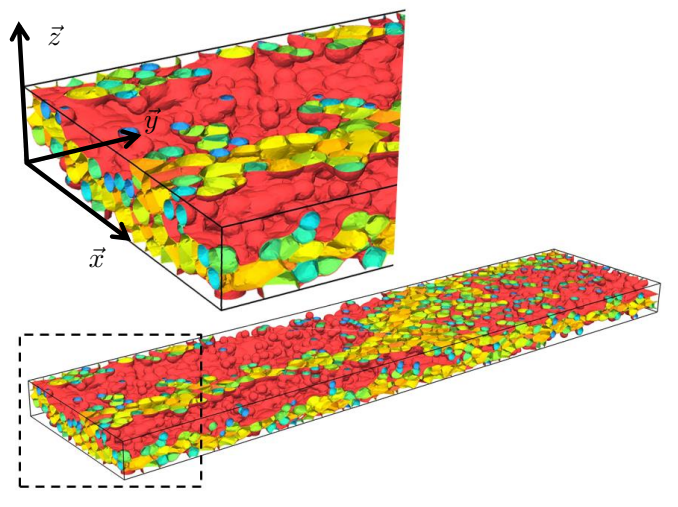

(b) $\varepsilon=1.5$

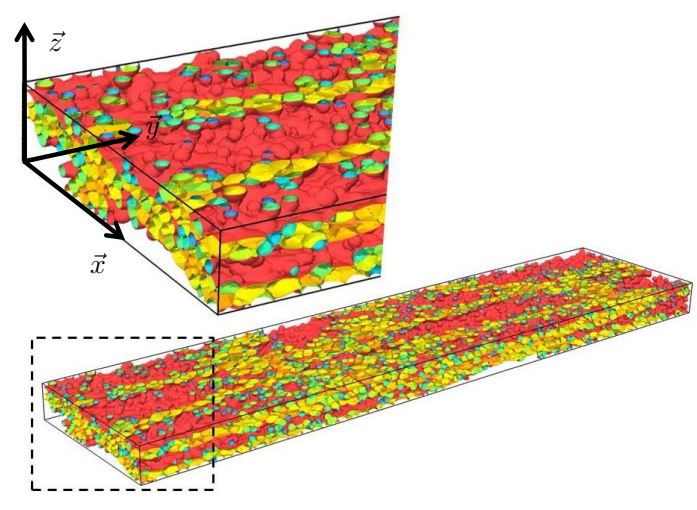

(d) $\varepsilon=1.5$

Figure 7: Two simulations using two different initial number of grains : 8 inital grains for a true deformation $\varepsilon=0$ (a) and $\varepsilon=1.5$ (b). 27 initial grains for a true deformation $\varepsilon=0$ (c) and $\varepsilon=1.5$ (d). The simulated process is a channel-die configuration at $1273^{\circ} \mathrm{K}$ and a strain rate of $0.01 \mathrm{~s}^{-1}$ during $150 \mathrm{~s}$. 
Furthermore, using 8 initial grains instead of 40 initial grains reduces the computational cost by a factor of 9, which is a non negligible time saving (see Fig. 8(d)). An initial number of 3 grains has also been tested. However, this small number of grains lead 315 to artefacts (important influence of the boundary conditions, a too small number of grains to respect precisely the input grain size distribution and an insufficient total grain boundary surface for the appearance of new grains after few time increments) and therefore to non-physical results, in particular concerning the distributions (see Fig. 8(e) and (f)). Thus, a minimum initial number of 8 grains will be chosen for all 320 the future simulations. 


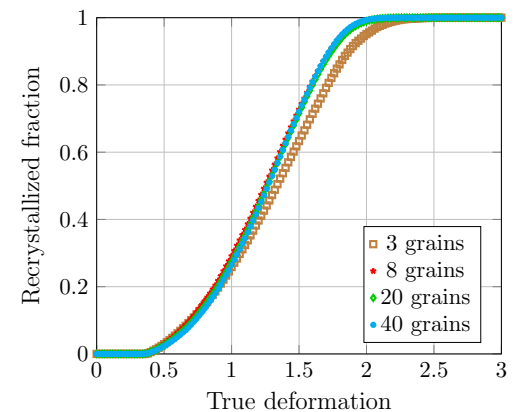

(a)

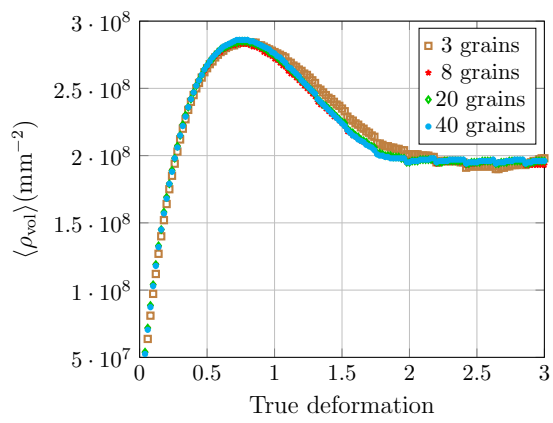

(c)

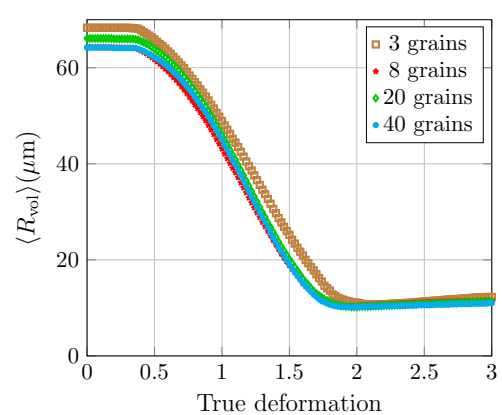

(b)

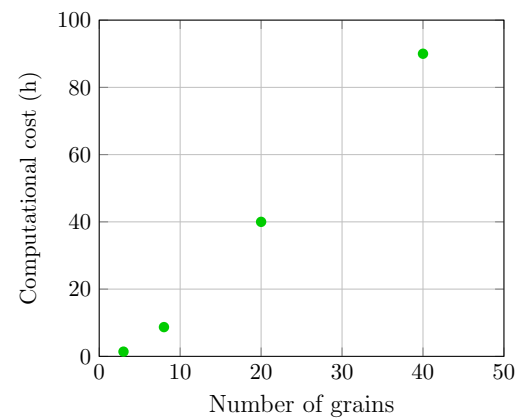

$(d)$

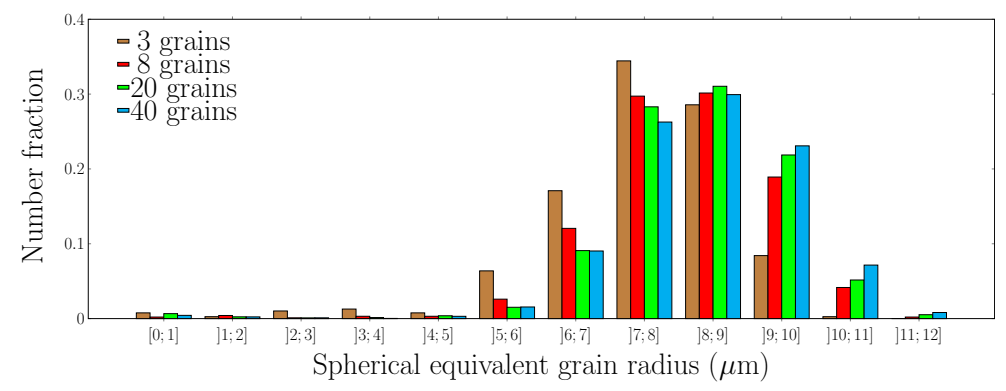

(e)

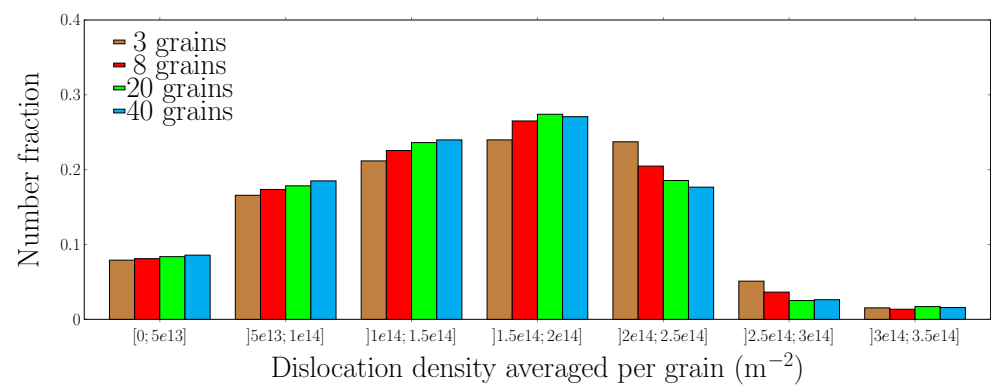

(f)

Figure 8: Sensitivity of the initial number of grains on polycrystal results: (a) recrystallized fraction, (b) mean grain radius, (c) mean dislocation density, (d) computational cost, (e) grain radius distributions for a true deformation $\varepsilon=1$ and (f) dislocation density distxibutions for a true deformation $\varepsilon=1$. The simulated process is a channel-die compression at $1273^{\circ} \mathrm{K}$, at a strain rate of $0.01 \mathrm{~s}^{-1}$ during $300 \mathrm{~s}$. The mean values are weighted by grain volume for a better representativeness of the curves at the onset of recrystallization. 


\section{Deformation step}

The strain hardening and recovery laws are directly related to the deformation step (see $\mathrm{Eq} 2$. Therefore a fixed time step is not enough to ensure convergence for any strain rate since the deformation step is given by the product between the time step and the strain rate. Thus, the idea of this section is to look for a deformation step that leads to converged results and then to use this ideal deformation step to deduce the associated time step.

According to the previous study, the initial number of grains is now fixed to 8 grains. The safety factor $\omega$ and the mesh size are still fixed and equal to 2 and $3 \mu \mathrm{m}$, respectively. To investigate the optimal deformation step, several simulations were done for four deformation steps $(2 \% ; 5 \% ; 10 \% ; 20 \%)$. The same results as the previous section (i.e. recrystallized fraction, mean grain radius, mean dislocation density, computational cost, grain radius distribution and dislocation density distribution) have been investigated in Fig. 9 When the deformation step decreases, results (recrystallized fraction, mean dislocation density, mean grain radius, grain radius distribution and dislocation density distribution) converge toward the same trends. The computational cost is reduced by a factor of about 2 between $2 \%$ and $5 \%$ of deformation step while keeping close results (see computational cost in Fig. 9 (d)). Thus a deformation step of $5 \%$ is chosen as a good compromise for all the future simulations.

\section{Critical nucleus radius}

The initial number of grains and deformation step are fixed to 8 and 5\% respectively, and the influence of the critical nucleus radius, more particularly the safety factor noted $\omega$ and used in Eq. 10, is thereafter investigated. Four different values for $\omega$ are tested $(1 ; 1.5 ; 2 ; 3)$ leading to the use of four different mesh sizes in order to respect the ratio $\xi \simeq 2$. The same results as the previous section (i.e. recrystallized fraction, mean grain radius, mean dislocation density, computational cost, grain radius distribution and dislocation density distribution) have been investigated in Fig. 10 First, it is observed that the final mean grain radius $\left\langle R_{\mathrm{vol}}\right\rangle$ and the grain radius distribution are highly influenced by the critical nucleus radius $r^{*}$. This observation is important because it means that the final mean grain radius can be directly influenced by the safety factor $\omega$. By 


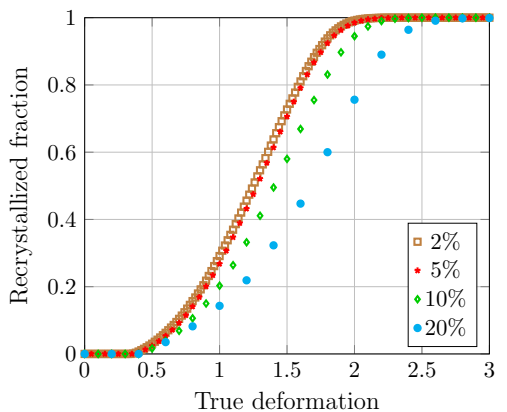

(a)

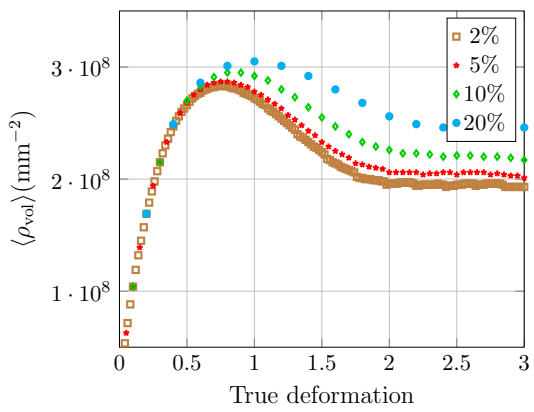

(c)

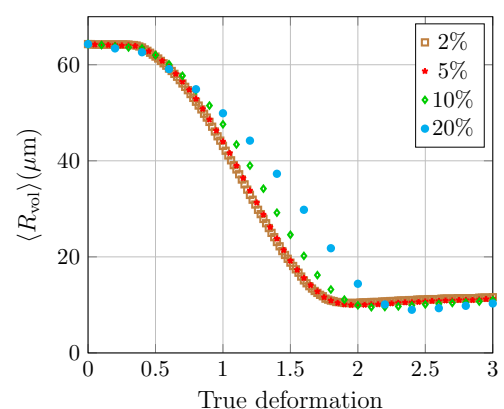

(b)

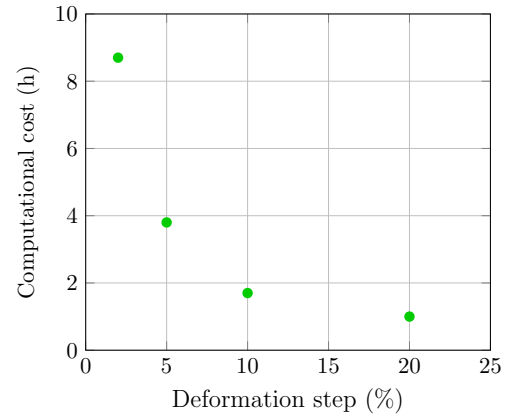

(d)

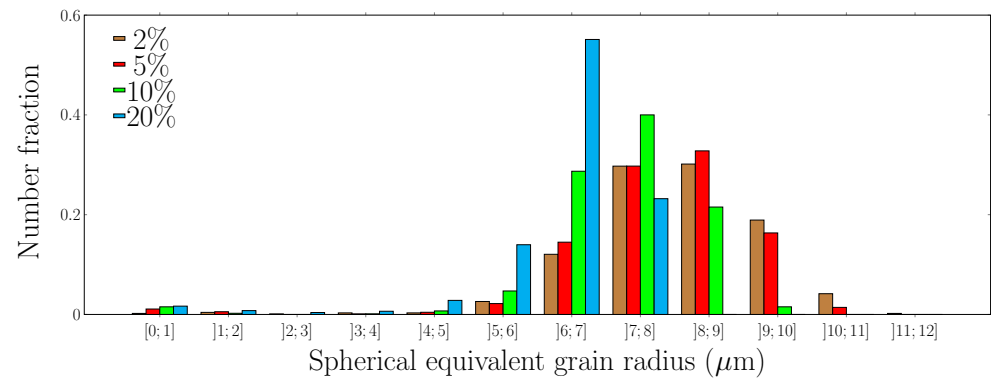

(a)

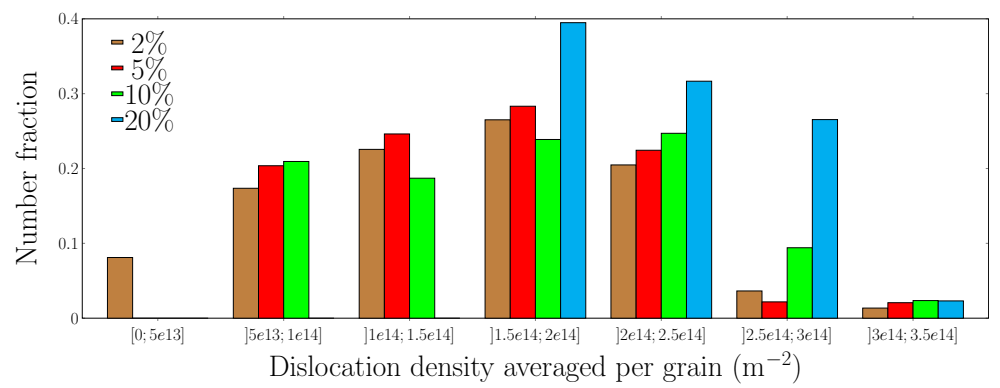

(b)

Figure 9: Sensitivity of the deformation step on polycrystal results: (a) recrystallized fraction, (b) mean grain radius, (c) mean dislocation density, (d) computational cost, (e) grain radius distributions for a true deformation $\varepsilon=1$ and (f) dislocation density distribytions for a true deformation $\varepsilon=1$. The simulated process is a channel-die compression at $1273^{\circ} \mathrm{K}$, at a strain rate of $0.01 \mathrm{~s}^{-1}$ during $300 \mathrm{~s}$. The mean values are weighted by grain volume for a better representativeness of the curves at the onset of recrystallization. 
observing the four other curves, it is clearly observed that a safety factor of 1 does not lead to converged results. This is due to the fact that a safety factor of 1 leads to a critical nucleus radius too low to counter capillarity effects exerted by neighboring grains and thus the new nuclei do not survive, which explains the observed kinetic of recrystallized fraction. In contrast, the two safety factors of 1.5 and 2 lead to a good convergence of all presented results. Finally, a safety factor of 3 does not lead to converged results in terms of recrystallized fraction and mean dislocation density because the critical nucleus radius is too large using this value and therefore results predicted by the model might be non-physical. Thus a safety factor of 1.5 is chosen as the optimal value for the future simulations. 


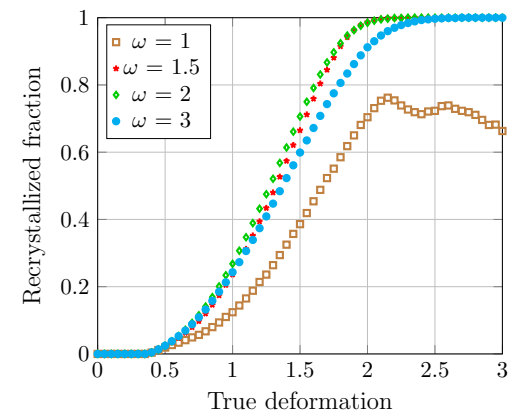

(a)

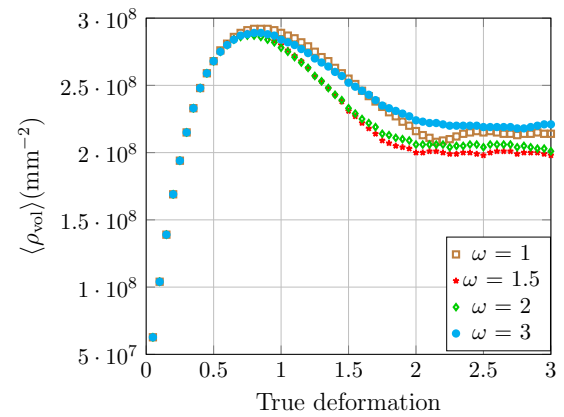

(c)

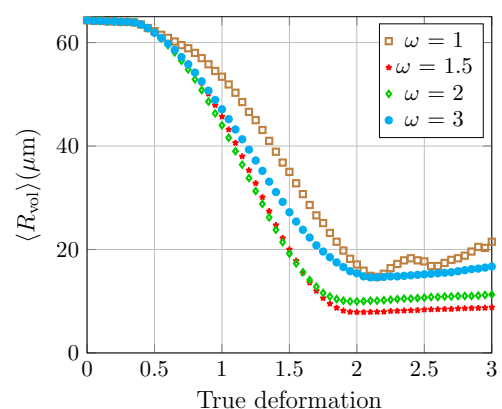

(b)

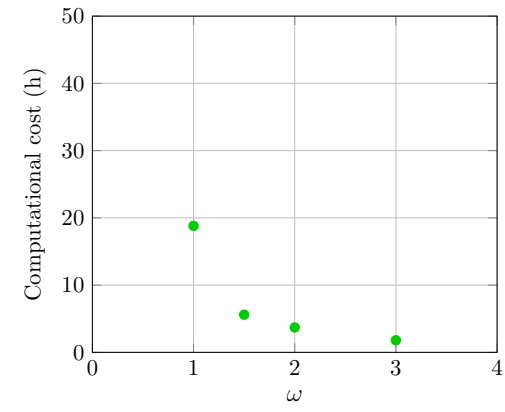

(d)

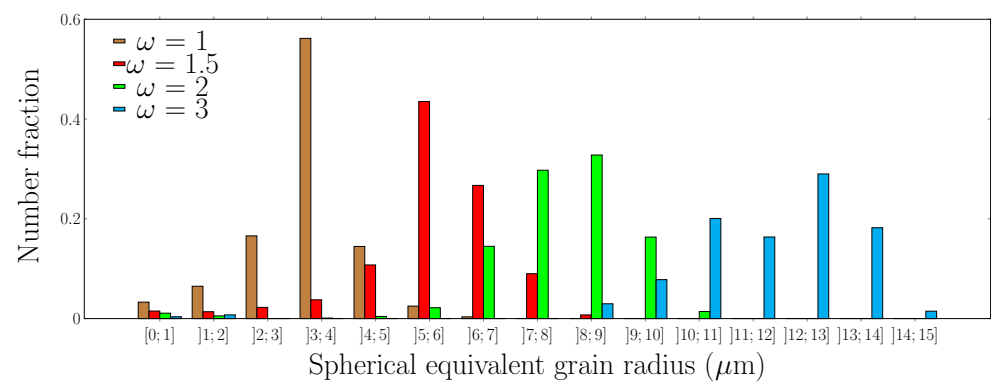

(e)

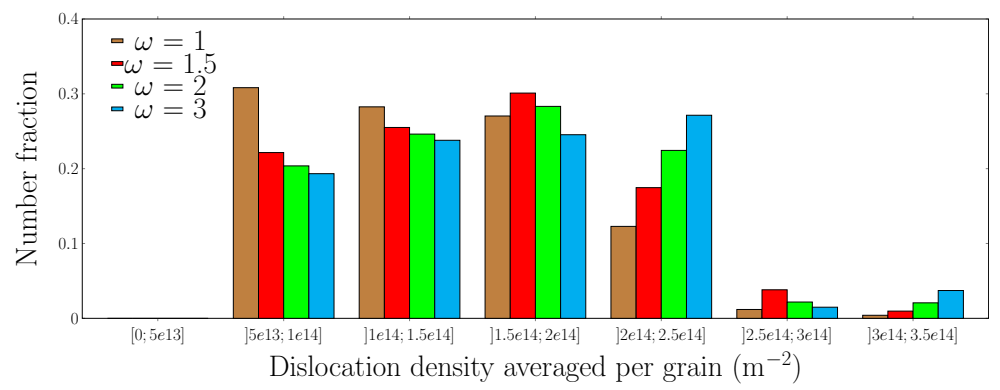

(f)

Figure 10: Sensitivity of the critical nucleus radius on polycrystal results: (a) recrystallized fraction, (b) mean grain radius, (c) mean dislocation density, (d) computational cost, (e) grain radius distributions for a true deformation $\varepsilon=1$ and (f) dislocation density disffibutions for a true deformation $\varepsilon=1$. The simulated process is a channel-die compression at $1273^{\circ} \mathrm{K}$, at a strain rate of $0.01 \mathrm{~s}^{-1}$ during $300 \mathrm{~s}$. The mean values are weighted by grain volume for a better representativeness of the curves at the onset of recrystallization. 


\subsection{Post-dynamic recrystallization}

\section{Time step}

The second convergence study concerns the ideal time step $(\Delta t)$ used for PDRX simulations. If the displacement of a grain boundary is too high during $\Delta t$, the kinetic cannot be correctly captured. Thus, a solution is to adapt $\Delta t$ as a function of a maximum displacement of grain boundaries. Let's consider the maximum displacement of an interface $d_{\max }$ in a microstructure between $t$ and $(t+\Delta t)$. This latter is given by the maximum velocity $v_{\max }$ in the microstructure between these two instants times the time increment $\Delta t$ :

$$
d_{\max }=v_{\max } \times \Delta t
$$

Furthermore, the highest value for $v_{\max }$ is reached if the smallest possible grain having the highest possible mean energy is surrounded by the largest possible grain having the lowest possible mean energy. Thus by using classical approximations for grain mean curvature, $v_{\max }$ is estimated as follow :

$$
v_{\max } \simeq M_{\mathrm{b}}\left(\frac{2 \gamma_{\mathrm{b}}}{\langle R\rangle}+\Delta E\right),
$$

375 ogy could be used by using in $\mathrm{Eq} 14$ the maximum values of these physical parameters. Finally by combining Eqs. 13 and 14 the max displacement $d_{\max }$ is estimated at each step time by :

$$
d_{\max } \simeq M_{\mathrm{b}}\left(\frac{2 \gamma_{\mathrm{b}}}{\langle R\rangle}+\Delta E\right) \times \Delta t .
$$

Then, it is necessary to find the ideal maximum displacement of grain boundaries in 380 order to determine the time step $\Delta t$ at each increment thanks to Eq. 15 In this study, we choose to defined the ideal maximum displacement $d_{\max }$ as a percentage of mesh size and this choice is validated in the following. 


\subsubsection{Validation of the criterion for time step}

To be representative of the PDRX mechanism, we assume four different simulations each representing a nucleus growing in a matrix (see Fig. 11. Each simulation is related to particular thermomechanical conditions (i.e. a couple of ${ }^{\circ} \mathrm{K}$ and $\dot{\varepsilon}$ ) leading to a given critical nucleus radius $r^{*}$ (by using Eq. 10) and leading to a maximum dislocation density field in the matrix computed by the ratio $K_{1} / K_{2}$ which is the steady state dislocation density according to Eq. 2 (since $K_{1}$ and $K_{2}$ depend on ${ }^{\circ} \mathrm{K}$ and $\dot{\varepsilon}$ ). Furthermore, a dislocation density field $\rho_{0}=1 \mathrm{e} 11 \mathrm{~m}^{-2}$, which is material dependant, is defined into the nucleus. We note $R_{\mathrm{P}}$ the ratio between the stored gradient energy $(\Delta E)$ and the capillarity effect $\left(2 \gamma_{\mathrm{b}} / r^{*}\right)$. For each case, the mesh size is chosen so as to keep the ratio $\xi$ between the nucleus radius and the mesh size approximatively equal

to 2 as demonstrated in the DRX sensitivity study of the previous section.

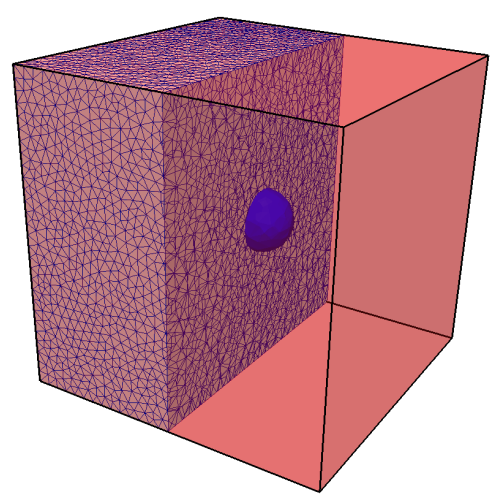

(a) $t=0 \mathrm{~s}$

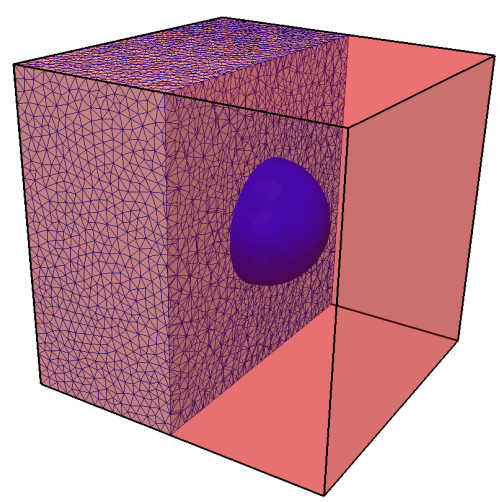

(b) $t=50 \mathrm{~s}$

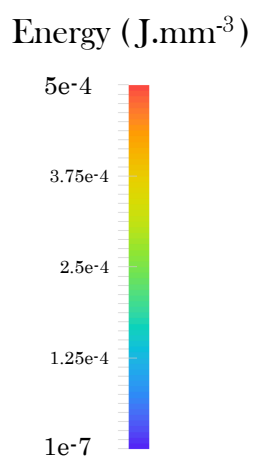

$1 e^{-7}$

Figure 11: Growth of a nucleus immersed in a matrix. The critical nucleus radius is equal to $5.2 \mu \mathrm{m}$. The energy in the nucleus and in the matrix are $1.47 \mathrm{e}-7 \mathrm{~J} . \mathrm{mm}^{-3}$ and $4.9 \mathrm{e}-4 \mathrm{~J} \cdot \mathrm{mm}^{-3}$, respectively.

Different percentages of mesh size from $10 \%$ to $40 \%$ are investigated as maximum displacement $d_{\max }$. In every cases, the evolution of the nucleus radius obtained by the numerical simulations is confronted to the analytical solution presented below :

$$
r^{(\mathrm{t}+\Delta \mathrm{t})}=r^{\mathrm{t}}+M_{\mathrm{b}} \Delta t\left(\Delta E-\frac{2 \gamma_{\mathrm{b}}}{r^{\mathrm{t}}}\right),
$$

The $\mathrm{L}^{2}$ errors between the analytical solution and the numerical results are plotted in 
Fig. 12 for each case corresponding to a particular couple of thermomechanical conditions.

First, it is observed in Fig. 12 that the lowest errors are obtained for cases where the gradient stored energy term is dominant (i.e. highest values of $R_{\mathrm{P}}$ ). This observation is logical and can be easily explained. The stored energy gradient term computed in the simulation is exact whereas the nucleus mean curvature is estimated by the Laplacian of the distance function and thus is mesh size dependant. Furthermore, as the mesh size is actually isotropic and non-refined around the interface, a non-negligible error can appear on the capillarity term in the simulation. Thus when the stored energy gradient is preponderant compared to the capillarity effects (i.e. high values of $R_{\mathrm{P}}$ ), the error made on the estimation of the mean curvature is of second order in the velocity term and lower errors are observed with respect to the analytical solution.

Furthermore for a given nucleus size, the errors are observed dependant on the percentages of mesh size displacement (see Fig. 12). It is observed that the minimum errors are globally obtained for the mesh size displacements of $10 \%, 20 \%$ or $30 \%$. For a very small displacement, the error due to the transport equation resolution of the LS method (see section 2.2.) can be relatively high compared to the small displacement of the interface, which can globally leads to inaccurate results. This aspect can explain that ${ }_{420}$ the $10 \%$-case is globally less accurate than the $20 \%$-case. For a large displacement (i.e. $40 \%$ of mesh size), the value of displacement seems too high during a single step time and thus the kinetic cannot be correctly captured. Indeed, our FE methodology remains of first order in time and important displacement during one time step can lead to nonphysical results. Finally, $10 \%, 20 \%$ or $30 \%$ of mesh sizes give better results than $40 \%$ of mesh size. However, for reasons of computational costs, we will retain a maximum displacement of mesh size $d_{\max }$ equal to $30 \%$ for the future PDRX simulations. 


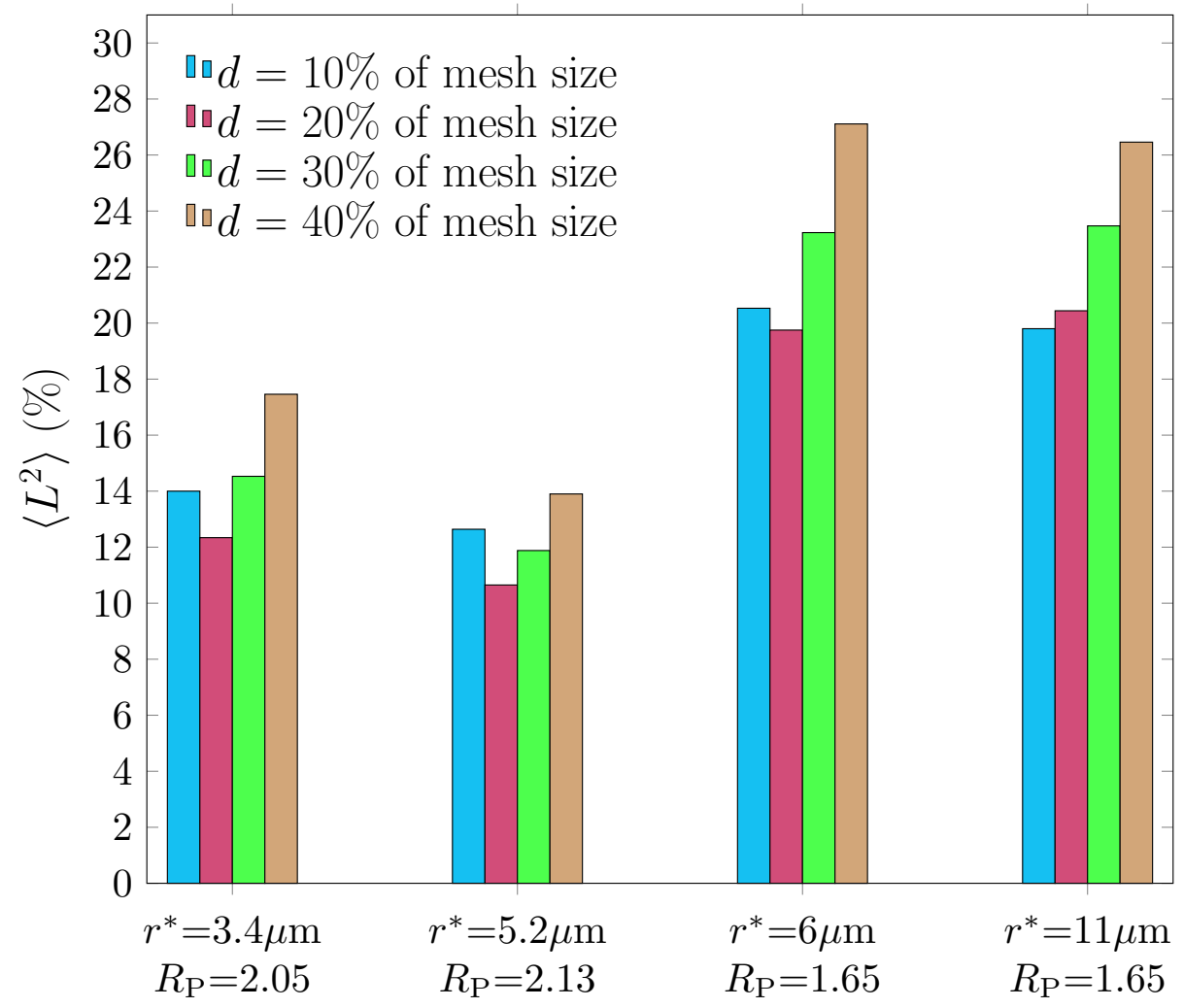

Figure 12: $L^{2}$ Error on the equivalent mean grain radius evolution between the full field simulation and the scheme defined by Eq. $16 r^{*}$ is the critical nucleus radius. $R_{\mathrm{p}}$ is the ratio between the velocity due to capillarity effects and the velocity due to the stored energy gradient. Each color corresponds to a specific displacement of mesh size performed at each increment of the model.

\section{Results and comparisons with a mean field model}

\subsection{Dynamic recrystallization}

This paper proposes a numerical framework based on phenomenological laws from an existing mean field model [13, 12] for nucleation criteria, hardening and recovery. However, contrary to mean field models, this model presents the advantage to fully describe, in time and space, the grain boundary network thanks to the level set approach for the modeling of grain boundary motion. Therefore, the idea of this section is to compare the above-mentioned mean field model of the literature [13, 12] with 
$R_{\mathrm{i}}$ and $\left\langle\rho_{\mathrm{i}}\right\rangle$ of these 8 classes are identical to the characteristics of the 8 initial grains considered in the full field simulation. The number of grains $N_{\mathrm{i}}$ in each class of the mean field model is chosen in order to respect the input distribution.

\begin{tabular}{llllllllll}
\hline & $K_{1}$ & $K_{2}$ & $K_{\mathrm{g}}$ & $K_{\mathrm{s}}$ & $M_{0}$ & $\delta(\dot{\varepsilon})$ & $\gamma_{\mathrm{b}}$ & $\tau$ & $Q_{\mathrm{m}}$ \\
\hline Unity & $\mathrm{m}^{-2}$ & & $\mathrm{~m} / \mathrm{s}$ & $\mathrm{s}^{-1}$ & $\mathrm{~m}^{4} /(\mathrm{J} . \mathrm{s})$ & & $\mathrm{J} / \mathrm{m}^{2}$ & $\mathrm{~J} / \mathrm{m}$ & $\mathrm{J} / \mathrm{mol}$ \\
\hline Values & $1.1 \mathrm{e} 15$ & 3.3 & $3.28 \mathrm{e}-8$ & 0.001 & $1.56 \mathrm{e}-1$ & 1.07 & 0.6 & $1.47 \mathrm{e}-9$ & $2.8 \mathrm{e} 5$ \\
\hline
\end{tabular}

Table 1: Set of parameters used in governing laws of both mean field and full field models for the considered 304L steel [13 57]. The deformation conditions associated to these parameters are the following : $\mathrm{T}=$ $1273^{\circ} \mathrm{K} ; \dot{\varepsilon}=0.01 \mathrm{~s}^{-1}$. 


\begin{tabular}{lllll}
\hline & $\langle R\rangle$ & $\sigma_{\mathrm{R}}$ & $\left\langle\rho_{\mathrm{v}}\right\rangle$ & $\sigma_{\rho}$ \\
\hline Unity & $\mu \mathrm{m}$ & $\mu \mathrm{m}$ & $\mathrm{m}^{-2}$ & $\mathrm{~m}^{-2}$ \\
Values & 60 & 10 & $6.5 \mathrm{e} 13$ & $1.25 \mathrm{e} 13$ \\
\hline
\end{tabular}

Table 2: Characteristics of the initial microstructure generated by the Voronoï tessellation algorithm and the associated energy field constant per grain generated from a Gaussian distribution.

The full field simulation was performed on 3 nodes of 24 CPU processors. Four different instants of the full field simulation are presented in Fig. 13 with a color code corresponding to the energy field constant per grain. The initial RVE measures $0.2 \mathrm{~mm}^{3}$ and is composed of 8 grains at the early stage of the simulation while around 9000 grains are present in the RVE at the end of the simulation. 


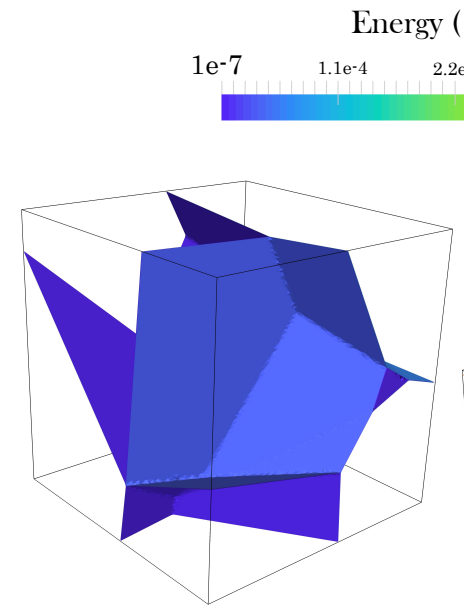

(a) $\varepsilon=0$

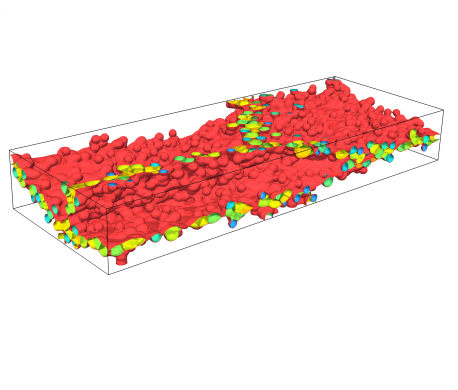

(b) $\varepsilon=1$

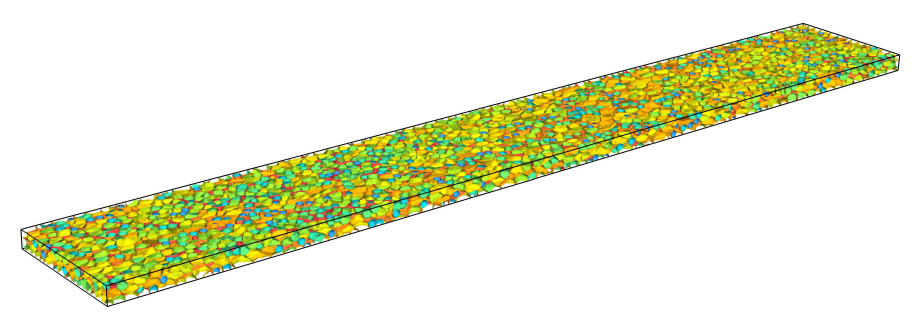

(c) $\varepsilon=2$

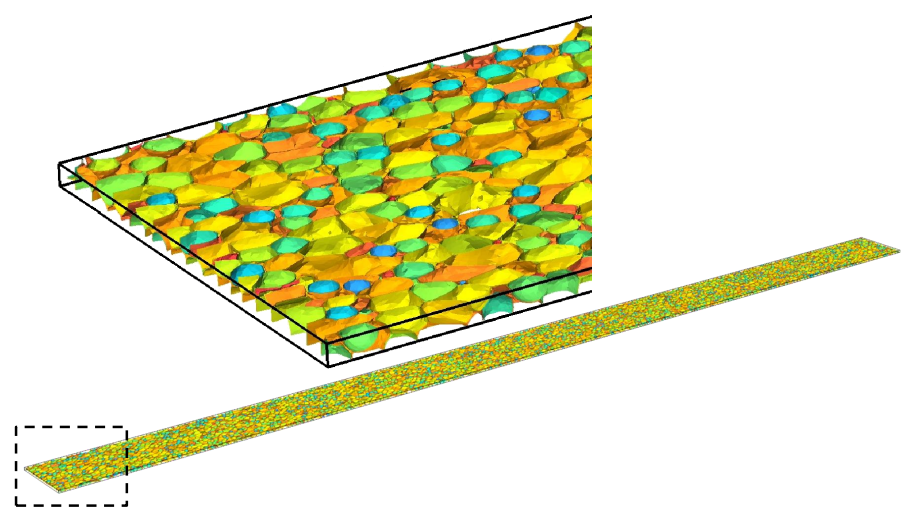

(d) $\varepsilon=3$

Figure 13: Four instants of a DRX simulation using the present full field model. The simulated process is a channel-die compression at $1273^{\circ} \mathrm{K}$ at a strain rate of $0.01 \mathrm{~s}^{-1}$ during $300 \mathrm{~s}$. Initial microstructure is composed of 8 grains while around 9000 grains are present at the end of deformation. Color code corresponds to the energy field constant per grain. 
Homogenized results in terms of mean dislocation density $\left\langle\rho_{\mathrm{vol}}\right\rangle$, mean grain radius $\langle R\rangle$, mean grain radius weighted by grain volume $\left\langle R_{\mathrm{vol}}\right\rangle$ and recrystallized fraction are confronted between the two models in Fig. 14. First it is observed that the evolutions of the mean grain radius $\langle R\rangle$ predicted by the two models are similar during all the process duration (see Fig. 14(c)) and final values are slightly higher than the critical nucleus radius $\left(\mathrm{r}^{*}=5.2 \mu \mathrm{m}\right)$. The decrease of $\langle R\rangle$ at the onset of recrystallization $(\varepsilon \simeq$ 0.4 ) is very fast and thus difficult to capture (see Fig. 14.(c)), thus the mean grain radius weighted by volume $\left\langle R_{\mathrm{vol}}\right\rangle$ is also plotted in Fig. $14(\mathrm{~d})$. During the first minutes of process (up to $\varepsilon=1$ ), the recrystallized fraction, $\left\langle R_{\mathrm{vol}}\right\rangle$ and $\left\langle\rho_{\mathrm{vol}}\right\rangle$ evolutions are quasi similar between the two models (see Fig. 14(a), (b), (d)). However, for a true deformation $\varepsilon>1$, the kinetics are always faster for the full field results, meaning that the number of nuclei appearing in the microstructure is always larger in the full field case. A first cause explaining this difference can be the evolution of grain boundary surfaces from equiaxed to ellipsoidal shape, increasing the surface of grain boundaries and consequently increasing the nucleation rate in the full field case (Eq. 11). Another cause explaining this difference is the quantity $\gamma_{\mathrm{RX}}$ presented in Fig. 14(f) and investigated in the following.

The quantities called $\gamma_{\mathrm{NR}}$ and $\gamma_{\mathrm{RX}}$ (see [12] for more information about the calculation of these quantities) represent respectively the mobile surface fraction of nonrecrystallized grains in contact with recrystallized grains and vice versa. These two quantities are used in the mean field model in order to calculate the grow rates of recrystallized and non-recrystallized grains. These quantities can be estimated from the full field simulation and compared with those computed in the mean field model. To estimate these quantities in the full field simulation, the surface fraction of each nonrecrystallized grain in contact with a recrystallized grain is calculated and averaged to get the quantity $\gamma_{\mathrm{NR}}$ and vice versa for $\gamma_{\mathrm{RX}}$. These values are measured at each instant of the full field simulation and are compared to the mean field model in Fig. 14.e) and (f). First, the increase of $\gamma_{\mathrm{NR}}$ observed in Fig. 14(e) is quiet similar for the two models. Furthermore, this evolution is very fast from the moment where nuclei appear $(\varepsilon \simeq 0.4)$.

${ }_{95}$ This fast increase is logical since nucleus mainly appear at grain boundaries, thus most 
of non-recrystallized grains are in contact with RX grains as soon as nucleation starts. The decrease of $\gamma_{R X}$ observed in Fig. 14(f) is perfectly similar for the two models up to a true deformation $\varepsilon \simeq 0.9$. After this instant, the decrease of $\gamma_{\mathrm{RX}}$ is much faster in the mean field model. The quantity $\gamma_{\mathrm{RX}}$ is used in the mean field model to calculate the growth rate of nuclei, thus a faster decrease of $\gamma_{R X}$ in the mean field case is a cause of the slower evolution of the recrystallized fraction, $\left\langle\rho_{\mathrm{vol}}\right\rangle$ and $\left\langle R_{\mathrm{vol}}\right\rangle$ predicted by the mean field model (see Fig. 14(a), (b) and (d)). 


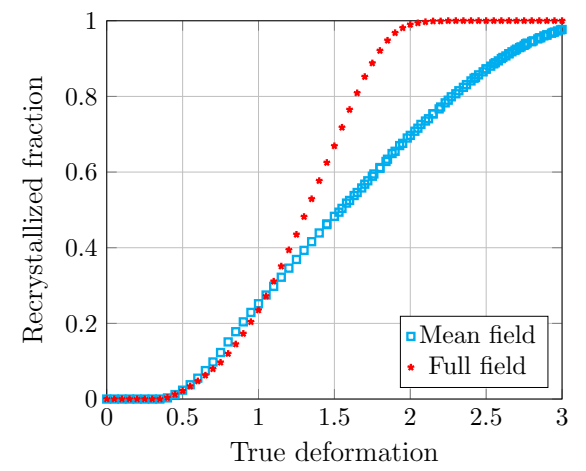

(a)

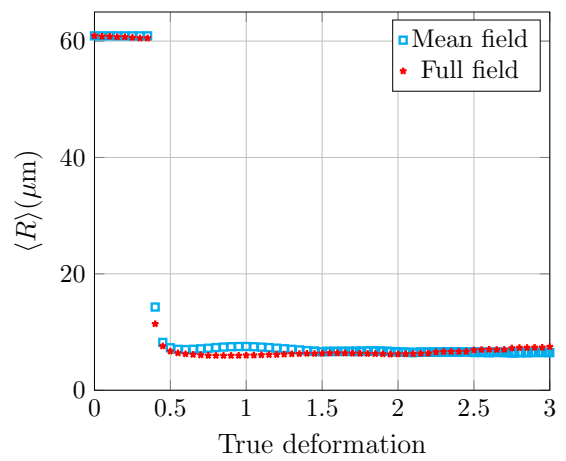

(c)

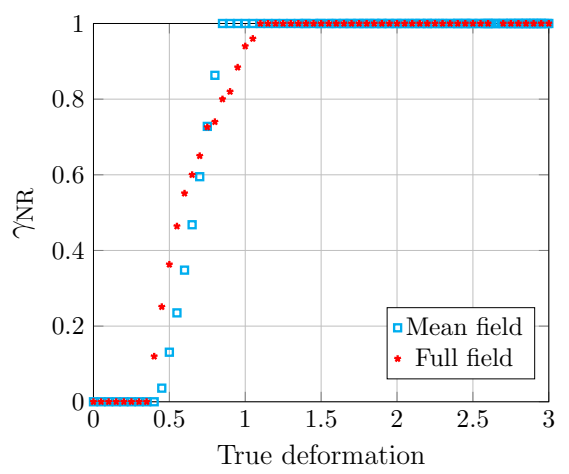

(e)

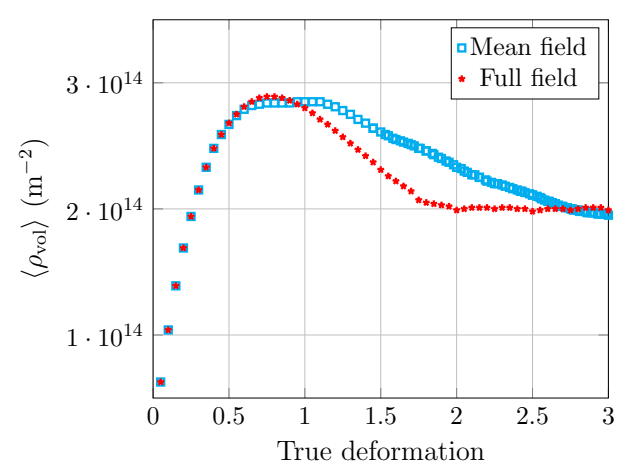

(b)

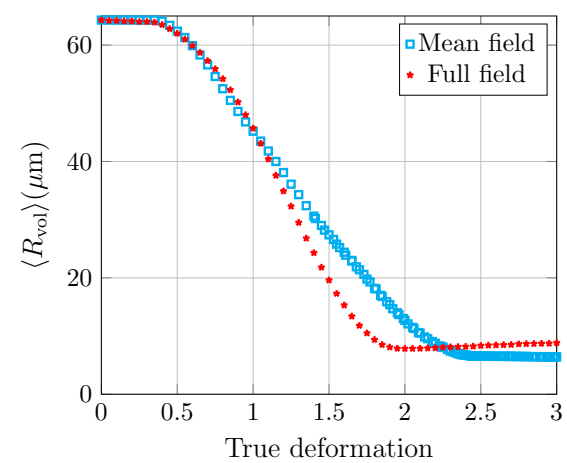

(d)

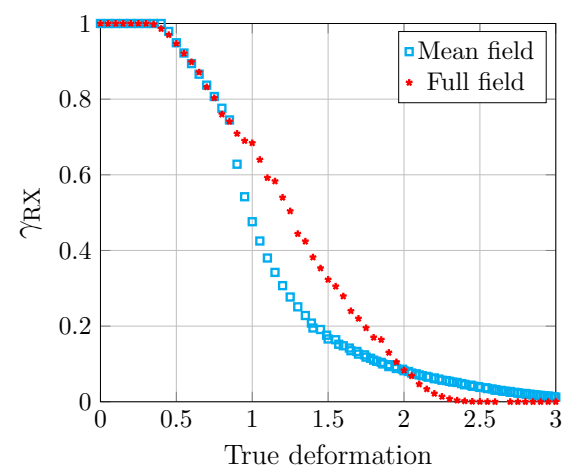

(f)

Figure 14: Comparisons of the macroscopic results obtained during a DRX process using the present full field model and a mean field model of the literature [12]. Compared results are: (a) recrystallized fraction weighted per grain volume, (b) mean dislocation density weighted by grain volume, (c) mean grain size, (d) mean grain size weighted by grain volume, (e) averaged surface fraction of non-recrystallized grains in contact with recrystallized grains and (d) averaged surface fraction of recrystallized grains in contact with non-recrystallized grains. The simulated process is a channel-die compression at $1273^{\circ} \mathrm{K}$, at a strain rate of $0.01 \mathrm{~s}^{-1}$ during $300 \mathrm{~s}$. 
Grain radius distributions and dislocation density distributions are also compared between the two models at four instants of the simulations (see Fig. 15 and Fig. 16. The grain radius distributions are represented in volume fraction since after few seconds of deformation, the number of nuclei is much higher than the number of initial grains. Thus a number fraction is not a discerning method to follow at the same time the small and large grains. The class width of histograms is $3 \mu \mathrm{m}$ for the grain radius distributions and $5 \mathrm{e} 13 \mathrm{~m}^{-2}$ for the dislocation density distributions.

510

The grain radius distributions predicted by the two models have still some similarities for a true deformation $\varepsilon=1$ (see Fig. 15. (b)). However, for a true deformation $\varepsilon=2$, a volume fraction of large grains is observed in the mean field simulation whereas in the full field simulation, the microstructure is fully recrystallized and these large grains have disappeared (see. Fig. 15 (c) and (d)). This difference is still due to the quantity $\gamma_{\mathrm{RX}}$ which decreases faster in the mean field case, leading to the fact that nuclei slowly growth at the expense of the non-recrystallized grains than in the full field case. Finally, a single narrow peak is observed in the mean field simulation for a true deformation $\varepsilon$ $=3$ at the steady-state regime whereas a larger peak is observed in the full field simulation (see Fig. 15.d)).

Concerning the dislocation density distributions, the shapes of the curves are close for the three instants $\varepsilon=1, \varepsilon=2$ and $\varepsilon=3$ of process (see Fig. 16 b), (c) and (d)). At each of these instants, the curves of the full field simulation have a more pronounced peak 525 than in the mean field simulation. 


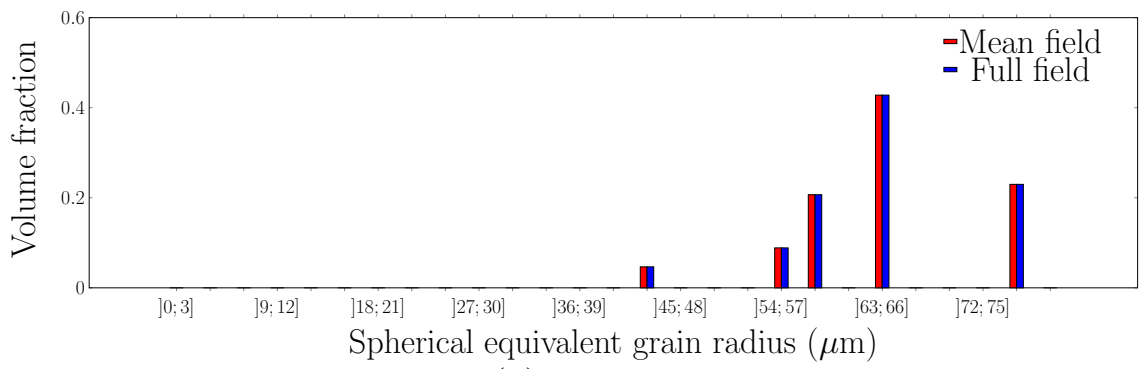

(a) $\varepsilon=0$

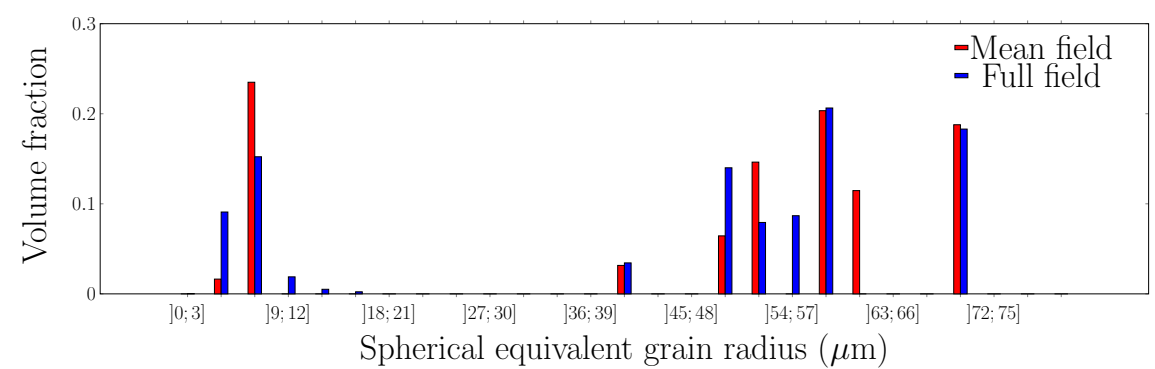

(b) $\varepsilon=1$

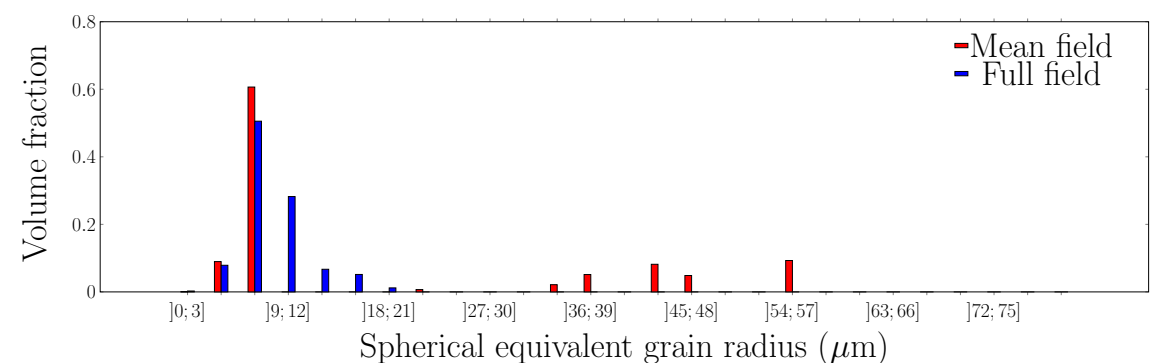

(c) $\varepsilon=2$

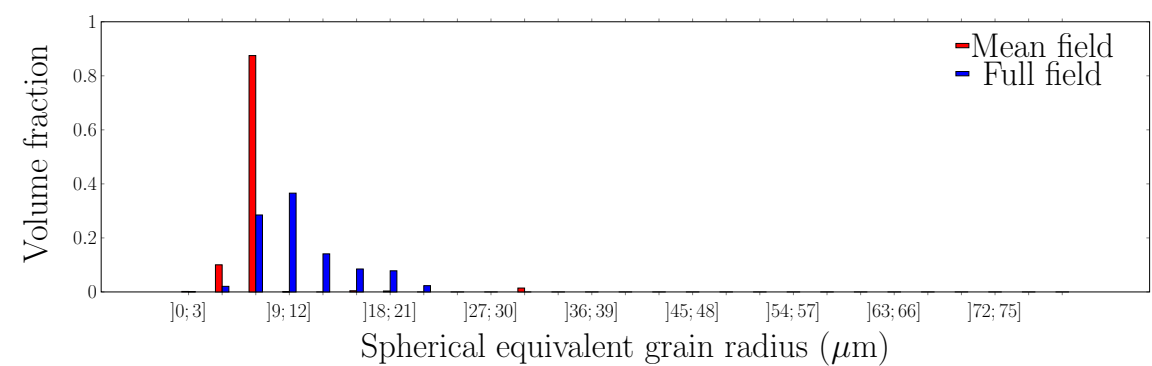

(d) $\varepsilon=3$

Figure 15: Comparisons, at different instants, of the grain radius distributions ((a) to (d)) obtained during a DRX process using the present full field model and a mean field model of the literature [12]. The simulated process is a channel-die compression at $1273^{\circ} \mathrm{K}$, at a strain rate of $0.01 \mathrm{~s}^{-1}$ during $300 \mathrm{~s}$. 


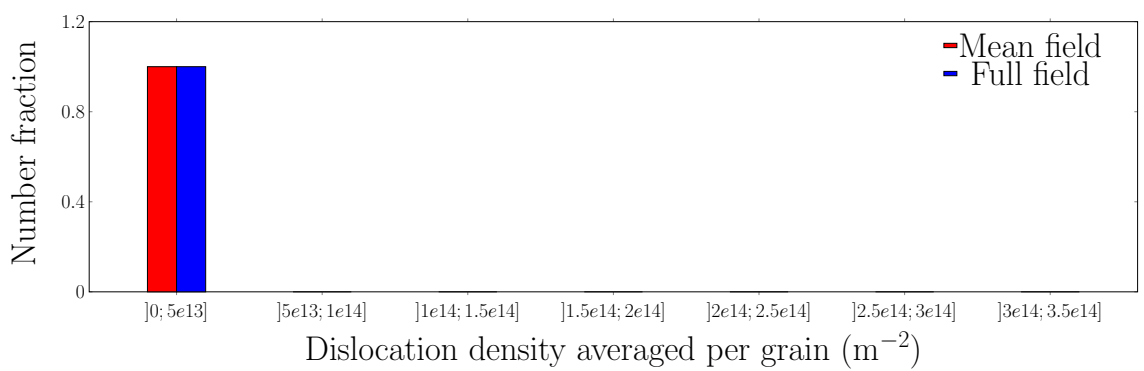

(a) $\varepsilon=0$

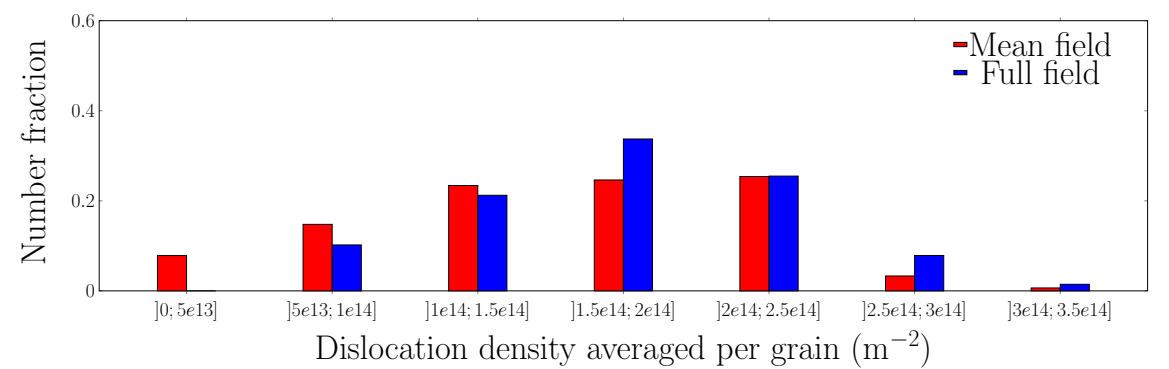

(b) $\varepsilon=1$

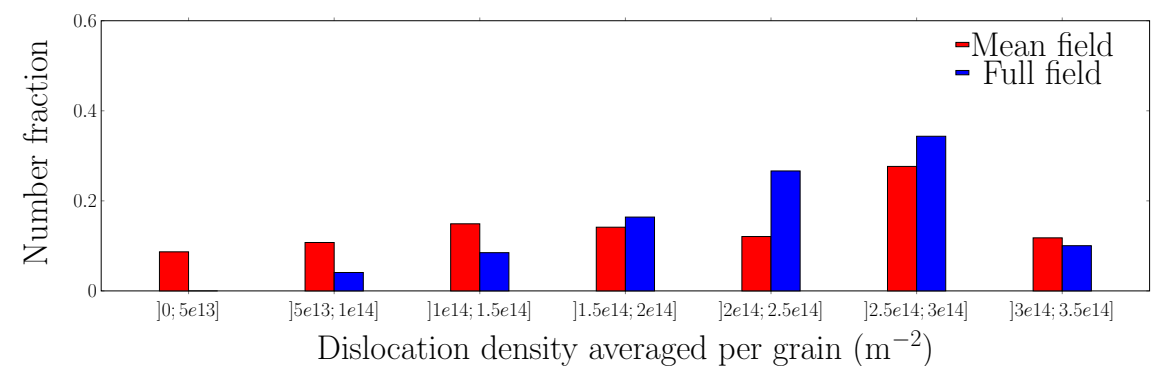

(c) $\varepsilon=2$

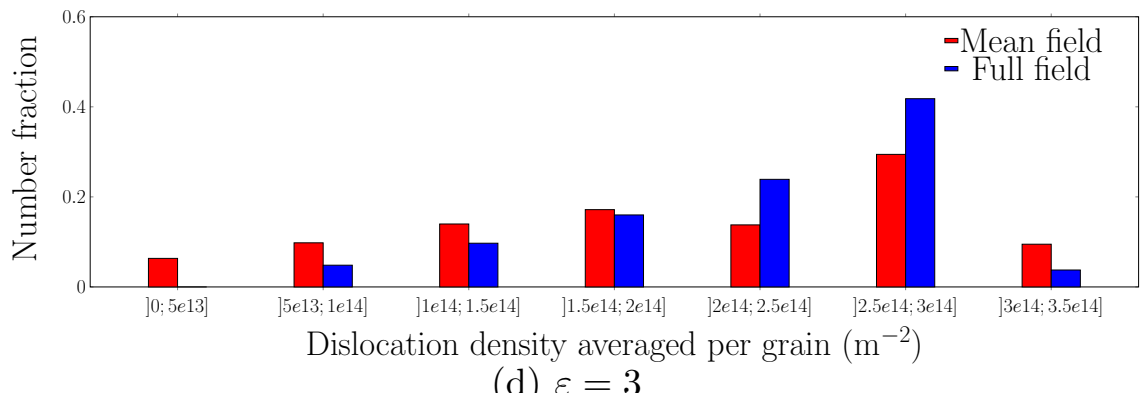

(d) $\varepsilon=3$

Figure 16: Comparisons, at different instants, of the dislocation density distributions ((a) to (d)) obtained during a DRX process using the present full field model and a mean field model of the literature [12]. The simulated process is a channel-die compression at $1273^{\circ} \mathrm{K}$, at a strain rate of $0.01 \mathrm{~s}^{-1}$ during $300 \mathrm{~s}$. 


\subsection{Post-dynamic recrystallization}

A PDRX simulation is then considered using the two models in order to compare the results. To avoid accumulating differences from DRX, the initial state of the microstructure used in both models corresponds to the final state microstructure obtained after a DRX simulation using the present full field model (see the subsection 5.1.). The PDRX process is simulated by considering a $304 \mathrm{~L}$ steel maintained at $1273^{\circ} \mathrm{K}$ during 25min. The first two minutes of PDRX are presented in Fig. 17 It is observed a large decrease of energy in the material during the first minutes of PDRX. After these few minutes, the preponderant mechanism is the capillarity driven grain growth mechanism

535 since the energy is low in the material and thus only the mean curvature of grains plays a primordial role on the pressure acting in the grain interfaces. 

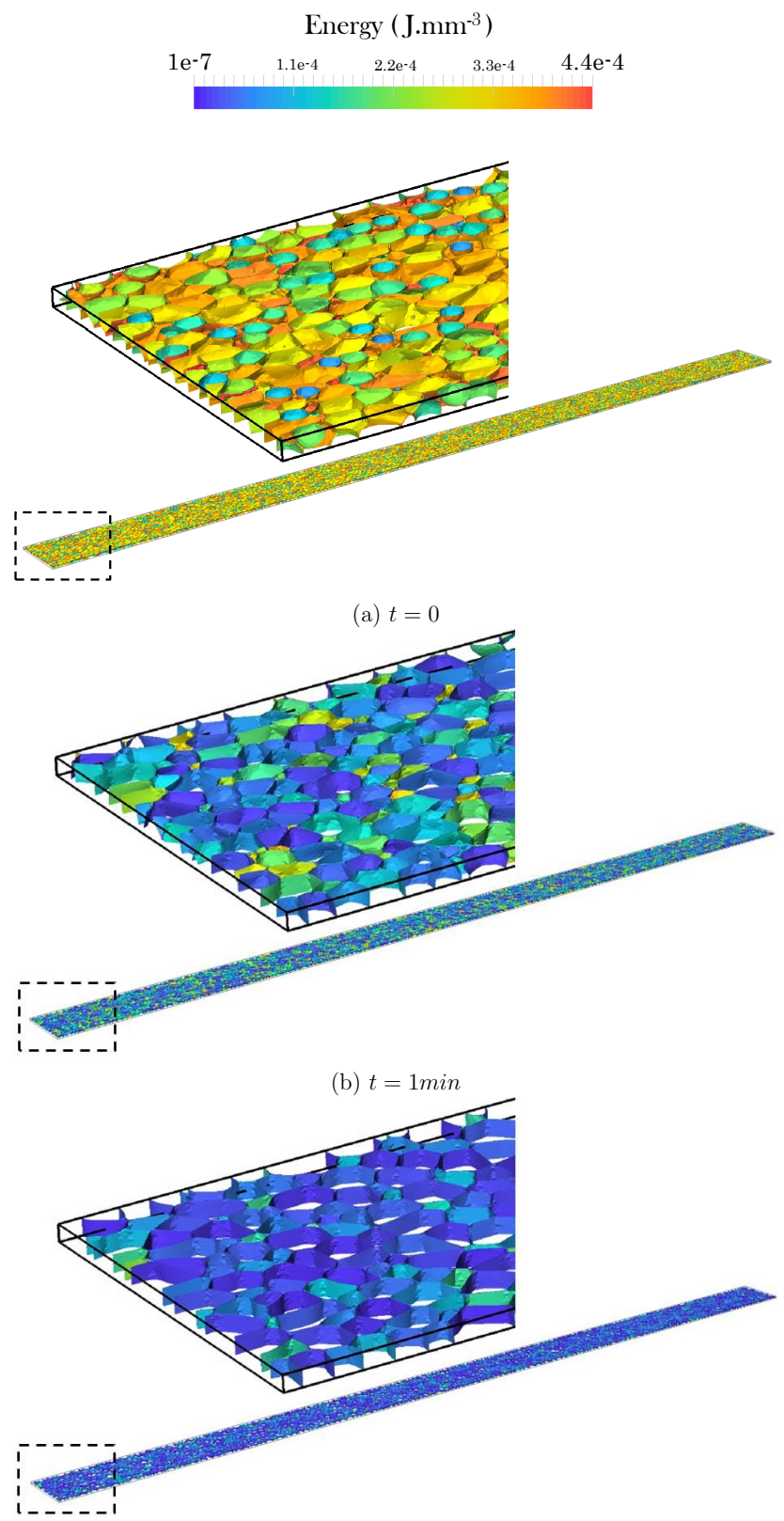

(c) $t=2 \min$

Figure 17: Three instants of the PDRX simulation using the present full field model. The simulated process is a heat treatment at $1273^{\circ} \mathrm{K}$ during $25 \mathrm{~min}$. Initial microstructure is composed of 9000 grains while around 600 grains are present at the end of the heat treatment. Color code corresponds to the mean energy per grain. 
Comparisons of mean dislocation density $\left\langle\rho_{\mathrm{vol}}\right\rangle$, mean grain radius $\langle R\rangle$ and mean grain radius weighted by grain volume $\left\langle R_{\mathrm{vol}}\right\rangle$ between the two models are presented in Fig. 18. The results obtained according to the two models are close. The evolutions of the curves are very pronounced at the early stage of the simulation since the energy is still high in the material. After few minutes, the energy is much more low in the material (see Fig. 17) and thus the grain boundary kinetic slows down, which is characterized by a slow evolution of $\langle R\rangle$ and $\left\langle R_{\mathrm{vol}}\right\rangle$ after this instant in Fig. 18 (b) and (c).

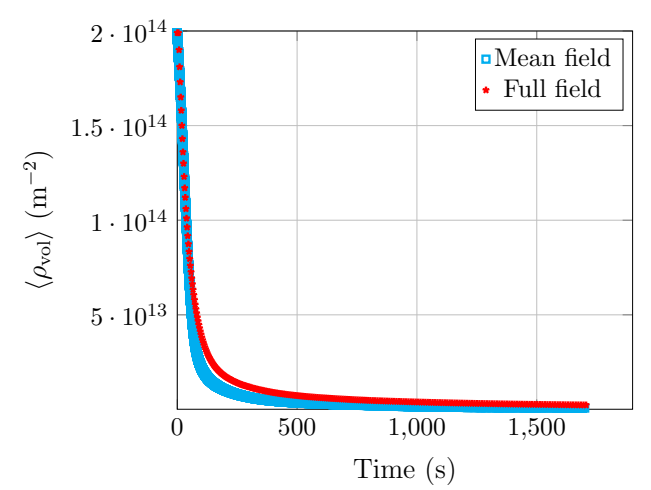

(a)

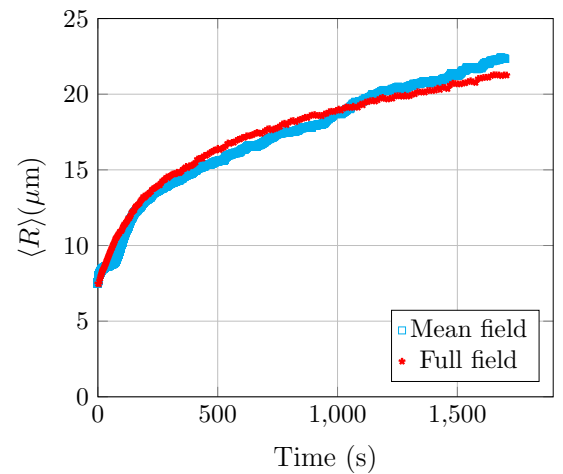

(b)

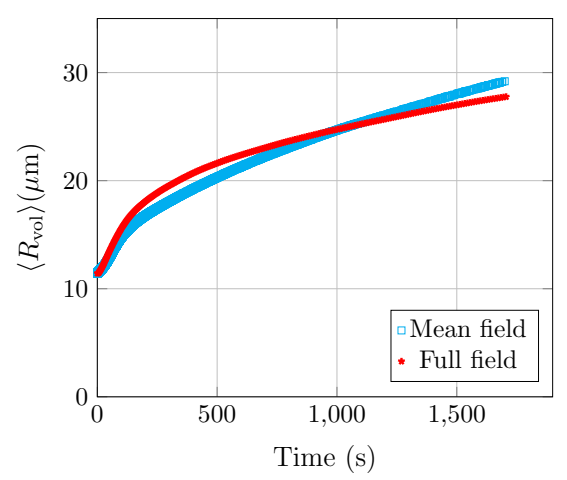

(c)

Figure 18: Comparisons of the macroscopic results obtained during a PDRX simulation using the present full field model and a mean field model of the literature [12]. Compared results are: (a) mean dislocation density weighted by grain volume, (b) mean grain size and (c) mean grain size weighted by grain volume. The simulated process is a heat treatment at $1273^{\circ} \mathrm{K}$ during $25 \mathrm{~min}$. 
${ }_{545}$ The grain radius distributions and dislocation density distributions are also presented and compared in Figs. 19 and 20. After 10 min of PDRX, the grain radius distribution is still very close between the two simulations (see Fig. 19.b)). However, after $20 \mathrm{~min}$ of PDRX, a single higher peak is appearing on the grain radius distribution of the mean field simulation (see Fig. 19. (c)) and this peak is still observed after 25min of PDRX ${ }_{550}$ (see Fig. 19 d)). In the full field simulation, this single higher peak is not observed during the simulation. Globally, it is observed that the grain radius distribution is poorly described by the mean field model. Concerning the dislocation density distributions, the results are identical in the two simulations since the energy quickly decreases in the material and thus after 10min of PDRX, all the grains have the same low energy and a single narrow peak is observed in the two models (see Fig. 20. 


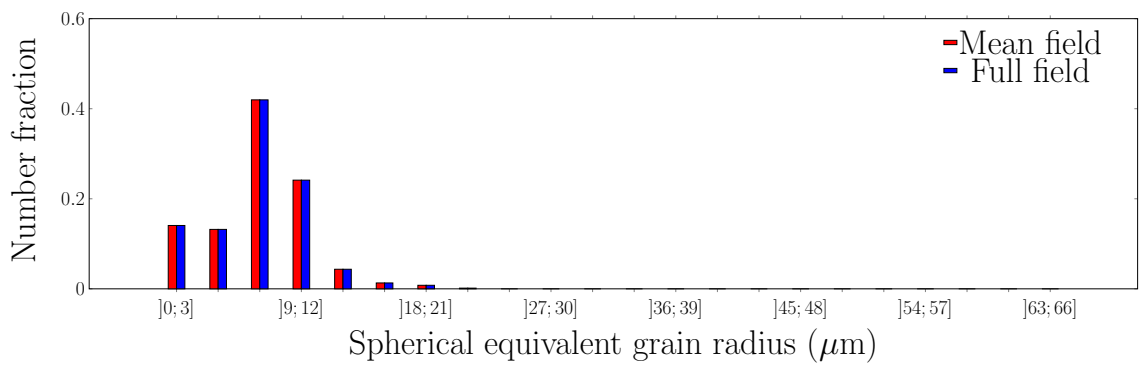

(a) $t=0 \mathrm{~min}$

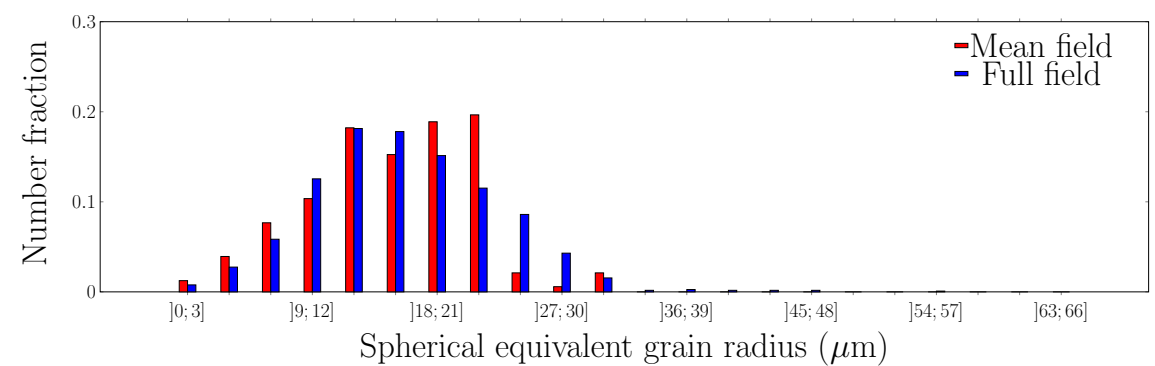

(b) $t=10 \mathrm{~min}$

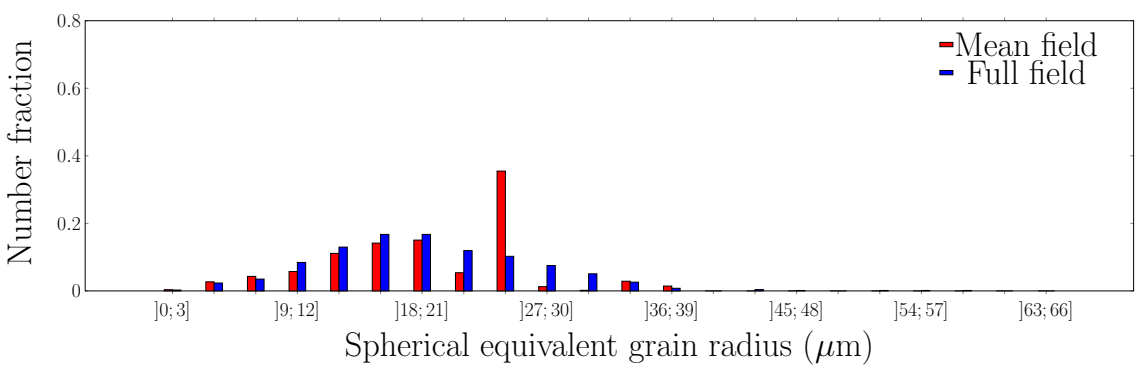

(c) $t=20 \mathrm{~min}$

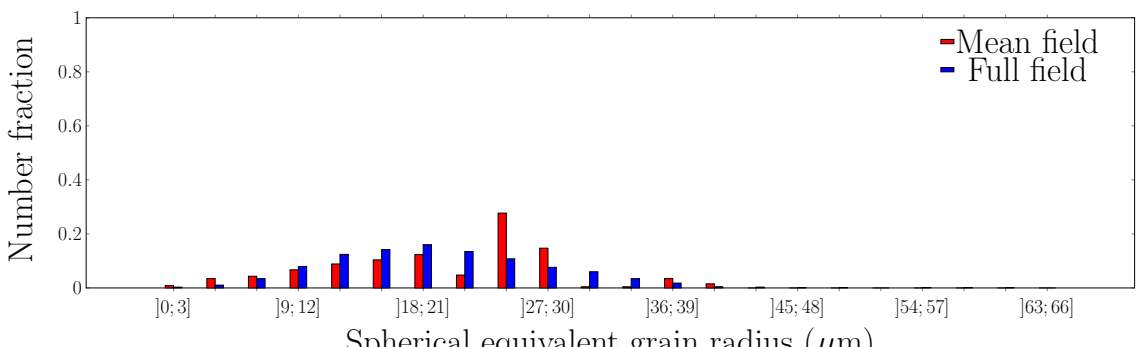

Spherical equivalent grain radius $(\mu \mathrm{m})$

(d) $t=25 \mathrm{~min}$

Figure 19: Comparisons, at different instants, of the grain radius distributions ((a) to (d)) obtained during a PDRX process using the present full field model and a mean field model of the literature [12]. The simulated process is a heat treatment at $1273^{\circ} \mathrm{K}$ during $25 \mathrm{~min}$. 


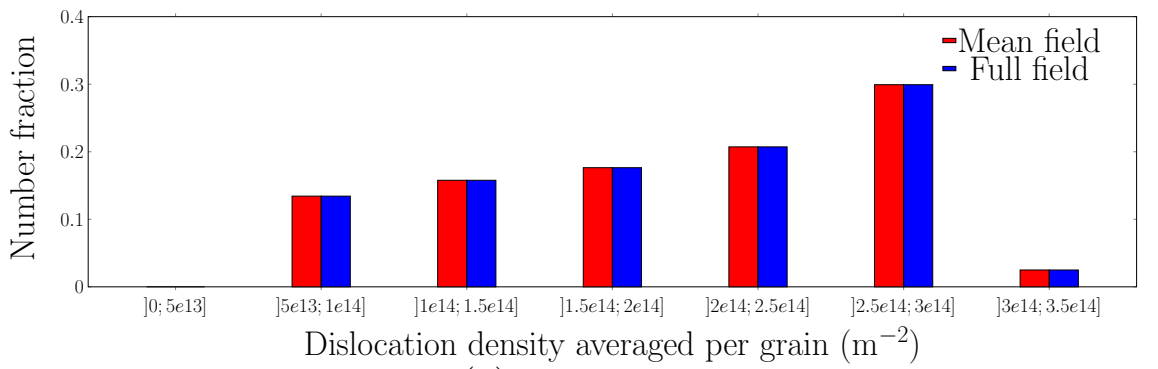

(a) $t=0 \mathrm{~min}$

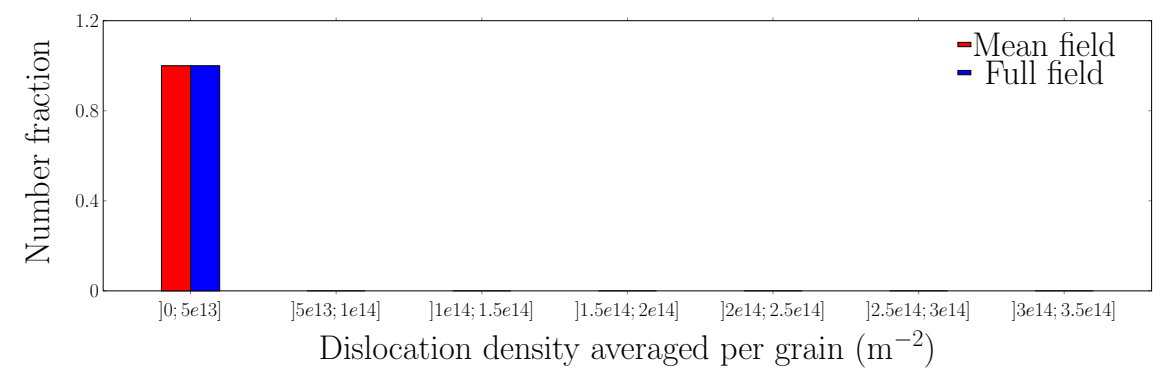

(b) $t=10 \mathrm{~min}$

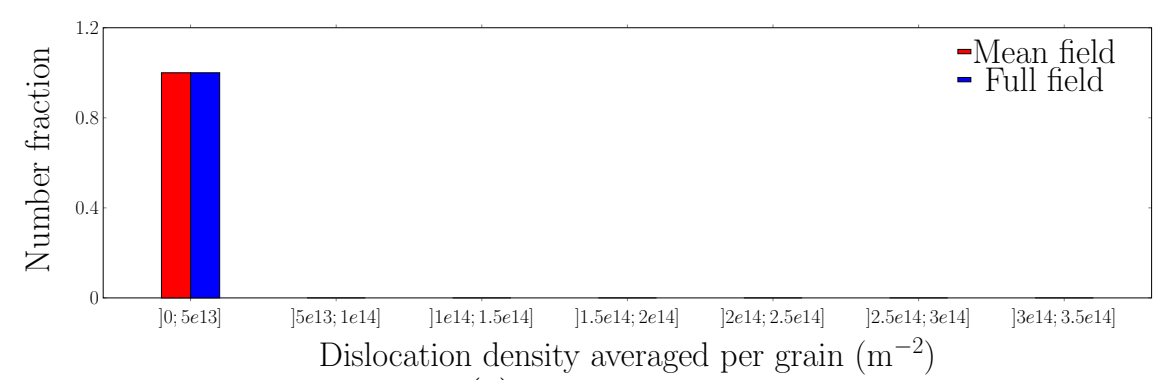

(c) $t=20 \mathrm{~min}$

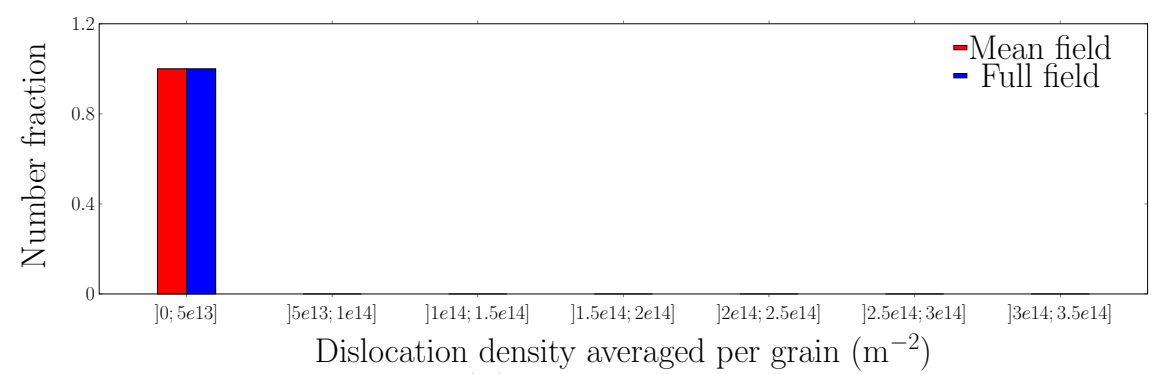

(d) $t=25 \mathrm{~min}$

Figure 20: Comparisons, at different instants, of the dislocation density distributions ((a) to (d)) obtained during a PDRX process using the present full field model and a mean field model of the literature [12]. The simulated process is a heat treatment at $1273^{\circ} \mathrm{K}$ during $25 \mathrm{~min}$. 


\section{Conclusions}

In the present work, a robust 3D model based on the LS method within a FE framework coupled to analytical laws has been presented to model the DRX and PDRX phenomena occurring during and after hot deformation of metals respectively. The advantages of the considered model are large : (i) the model enables to perform 3D simulations; (ii) the mechanisms of strain hardening, static and dynamic recovery are simulated thanks to phenomenological laws coming from a pre-existing mean field model [12, 13], which is not as accurate as with a crystal plasticity finite element formulation, but considerably reduces the computational costs of the simulations; (iii) the considered 3D model is able to describe the grain boundary network using the level-set method, even at large deformation (for instance $\varepsilon>0.2$ ) in order to reproduce industrial processes and (iv) a sensitivity study has largely minimized computational costs, allowing the integration of the model in an industrial context through the DIGIMU ${ }^{\circledR}$ software package.

Results of DRX and PDRX simulations using this new numerical framework were also confronted with the prediction of the above-mentioned mean field model [12, 13]. Results have shown that the mean field model provides a poor description of the recrystallized fraction and grain size distributions during hot deformation. This difference reinforces the idea that the proposed full field model is much more powerful than a mean field model since it explicitly takes the grain boundary network into account. After hot deformation, the whole results predicted by the mean field model, except the grain size distribution, are close to the full field predictions.

Future investigations will aim to (i) improve the mean field model by tackling some considered assumptions; (ii) validate the proposed full field formalism thanks to experimental results and (iii) confront this model to a crystal plasticity-based DRX full field model.

\section{Acknowledgements}

The authors thank the AREVA NP, ArcelorMittal, ASCOMETAL, AUBERT \& DUVAL, CEA, SAFRAN, TIMET and TRANSVALOR companies and the ANR for 
Prof. R. Logé for the fruitful discussions concerning the mean field model and $\gamma_{\mathrm{NR}} / \gamma_{\mathrm{RX}}$ equations.

\section{Appendices}

\section{A. Representation of the grain boundary network}

The Voronoï method consists in generating a diagram composed of a set of $N$ Voronoï nuclei $\left(S_{\mathrm{i}}\right)$. Then, a single Voronoï cells $V_{\mathrm{i}}$ per nucleus is defined following this rule: each Voronoï cell is composed of all points closer to $S_{\mathrm{i}}$ than to any other nuclei. However, the Voronoï method cannot respect a given grain size distribution. Thus, a second method called Laguerre-Voronoï can be used. This method consists in generating a diagram where the locations of the cells faces are constrained by a given non-intersecting spherical packing. Thus, the diagram is composed of $N$ seeds each with a weight $\left(S_{\mathrm{i}}, r_{\mathrm{i}}\right)$. Then, a single Laguerre-Voronoï $L_{\mathrm{i}}$ is created per seed following this new rule: each Laguerre-Voronoï cell is composed of all points closer to $S_{\mathrm{i}}$ than to any other nuclei, via the power distance. Where the power distance from $S_{\mathrm{i}}$ to $\mathrm{x}$ is defined by $d\left(x, S_{\mathrm{i}}\right)^{2}-r_{\mathrm{i}}^{2}$.

\section{B. Level set method for grain boundaries description}

A LS function $\psi$ is defined over a domain $\Omega$ as the signed distance function to the interface $\Gamma$ of a sub-domain $\mathrm{G}$ of $\Omega$. The values of $\psi$ are calculated at each interpolation point (node in the considered P1 formulation) and the sign convention states $\psi \geq 0$ inside $\mathrm{G}$ and $\psi \leq 0$ outside :

$$
\left\{\begin{array}{c}
\psi(x, t)= \pm d(x, \Gamma), x \in \Omega, \\
\Gamma(t)=\{x \in \Omega, \psi(x, t)=0\},
\end{array}\right.
$$

where $d$ corresponds to the Euclidean distance. The equations to evaluate analytically the LS functions of a Voronoï or a Laguerre-Voronoï tessellation are detailed in [49]. Generally, a single LS function is able to describe a single grain boundary leading to a number of LS functions $N$ equal to the number of grains $N_{\mathrm{g}}$ in the microstructure 
( $N=N_{\mathrm{g}}$ ). To limit the number of LS functions and thus the computational cost, a coloring/recoloring technique has recently been developed and applied to this model [31]. This technique aims to gather several grain boundaries in each LS function, leading to a number of LS functions significantly lower than the number of grains $\left(N \ll N_{\mathrm{g}}\right)$ during all the simulation.

\section{Grain boundary migration kinetic}

To simulate the kinetic of grain boundaries, each LS interface is displaced during the simulation according to a given velocity field $\vec{v}$ by solving a transport equation :

$$
\left\{\begin{array}{l}
\frac{\partial \psi(x, t)}{\partial t}+\vec{v} \cdot \vec{\nabla} \psi(x, t)=0, \\
\psi(x, t=0)=\psi^{0}(x),
\end{array}\right.
$$

Where $\psi^{0}(x)$ is the LS interface at $\mathrm{t}=0$ s. The velocity is assumed to be the contribution of two terms :

$$
\vec{v}=\vec{v}_{\mathrm{c}}+\vec{v}_{\mathrm{e}}
$$

where $\vec{v}_{\mathrm{c}}$ and $\vec{v}_{\mathrm{e}}$ are respectively the velocities due to capillarity effects and stored energy gradients expressed as follow :

$$
\begin{aligned}
& \vec{v}_{\mathrm{e}}=M_{\mathrm{b}} \delta(\dot{\varepsilon}) \Delta E \vec{\nabla} \psi, \\
& \vec{v}_{\mathrm{c}}=-M_{\mathrm{b}} \gamma_{\mathrm{b}} \Delta \psi \vec{\nabla} \psi,
\end{aligned}
$$

where $M_{\mathrm{b}}$ is the grain boundary mobility, $\delta(\dot{\varepsilon})$ is a strain rate dependant parameter detailed in the following, $\Delta E$ is the stored energy gradient across the interface and $\gamma_{b}$ is the grain boundary energy. These descriptions of the different kinetic terms are correct ${ }_{625}$ if the LS function $\psi$ is a distance function ( i.e. $\|\nabla \psi\| \equiv 1$ ) at least inside a thin layer $|\psi| \leq L$ around the interface. 
The grain boundary mobility $M_{\mathrm{b}}$, appearing in Eqs. C.3 and C.4 can be written as a function of temperature :

$$
M_{\mathrm{b}}=M_{0}(\mathrm{~T}) \exp \left(\frac{-Q_{\mathrm{m}}}{R T}\right) \text {, }
$$

The interfaces of every grains belonging to the $\mathrm{i}^{\text {th }} \mathrm{LS}$ function $\left(\psi_{i}\right)$ is thus implicitly given at each time step by the equation $\psi_{i}(t, x)=0$. A major drawback of the LS method is the loss of metric properties after the resolution of Eq. C.6 Indeed, even 


\section{References}

[1] D. Li, Q. Guo, S. Guo, H. Peng, Z. Wu, The microstructure evolution and nu1 cleation mechanisms of dynamic recrystallization in hot-deformed Inconel 625

a superalloy, Materials and Design 32 (2) (2011) 696-705. doi:10.1016/j. matdes.2010.07.040

URL http://dx.doi.org/10.1016/j.matdes .2010.07.040

[2] H. Jiang, L. Yang, J. Dong, M. Zhang, Z. Yao, The recrystallization model and microstructure prediction of alloy 690 during hot deformation, Materials and Design 104 (2016) 162-173. doi:10.1016/j.matdes .2016.05.033

670 URL http://dx.doi.org/10.1016/j.matdes.2016.05.033

[ [3] Z. Wan, Y. Sun, L. Hu, H. Yu, Experimental study and numerical simulation of dynamic recrystallization behavior of TiAl-based alloy, Materials \& Design 122 (2017) 11-20. doi:10.1016/j.matdes.2017.02.088.

URL http://dx.doi.org/10.1016/j.matdes.2017.02.088

${ }_{675}$ [4] K. Huang, R. Logé, A review on dynamic recrystallization phenomena in metallic materials, Materials \& Design 111 (2016) 548-574. doi:10.1016/j.matdes.2016.09.012

1 URL http://linkinghub.elsevier.com/retrieve/pii/ S0264127516311753

${ }_{680}$ [5] X. G. Fan, H. Yang, P. F. Gao, R. Zuo, P. H. Lei, The role of dynamic and post a dynamic recrystallization on microstructure refinement in primary working of a 
coarse grained two-phase titanium alloy, Journal of Materials Processing Technology 234 (2016) 290-299. doi:10.1016/j .jmatprotec. 2016.03.031.

URL http://dx.doi.org/10.1016/j.jmatprotec.2016.03.031

[11] D. G. Cram, H. S. Zurob, Y. J. M. Brechet, C. R. Hutchinson, Modelling discontinuous dynamic recrystallization using a physically based model for nucleation,

07.024

URL http://dx.doi.org/10.1016/j.actamat.2009.07.024 
[12] P. Bernard, S. Bag, K. Huang, R. Logé, A two-site mean field model of discontinuous dynamic recrystallization, Materials Science and Engineering: A 528 (24) (2011) 7357-7367. doi:10.1016/j.msea.2011.06.023 S0921509311006757

[13] O. Beltran, K. Huang, R. Logé, A mean field model of dynamic a and post-dynamic recrystallization predicting kinetics, grain size and flow stress Computational Materials Science 102 (2015) 293-303. doi:10.1016/j.commatsci.2015.02.043.

1 URL http://linkinghub.elsevier.com/retrieve/pii/ S0927025615001445

[14] L. Maire, B. Scholtes, C. Moussa, N. Bozzolo, D. Pino Muñoz, M. Bernacki, 1 Improvement of 3D mean field models for capillarity-driven grain growth based on full field simulations, Journal of Materials Science 51 (24) (2016) 1097010981. doi:10.1007/s10853-016-0309-6. URL http://link .springer . com/10.1007/s10853-016-0309-6

[15] H. Hallberg, Approaches to Modeling of Recrystallization, Metals 1 (1) (2011) 16-48. doi:10.3390/met1010016. URL http: //www .mdpi . com/2075-4701/1/1/16/

[16] A. D. Rollett, D. J. Srolovitz, M. P. Anderson, Simulation and theory of abnormal grain growth-anisotropic grain boundary energies and mobilities, Acta Metallurgica 37 (4) (1989) 1227-1240. doi:10.1016/0001-6160(89)90117-X.

[17] A. D. Rollett, D. Raabe, A hybrid model for mesoscopic simulation of recrystallization, Computational Materials Science 21 (1) (2001) 69-78. doi:10.1016/S0927-0256(00)00216-0.

735 URL http://wWw.sciencedirect.com/science/article/pii/ S0927025600002160

[18] D. Raabe, Introduction of a scalable three-dimensional cellular automaton with 
[19] D. Raabe, Cellular Automata in Materials Science with Particular Reference to Recrystallization Simulation, Annual Review of Materials Research 32 (1)

[20] L. Barrales Mora, G. Gottstein, L. Shvindlerman, Three-dimensional grain growth: Analytical approaches and computer simulations, Acta Materialia 56 (20)

[21] C. E. Krill, L. Q. Chen, Computer simulation of 3-D grain growth using a phase-field model, Acta Materialia 50 (12) (2002) 3059-3075. doi:10.1016/S1359-6454(02)00084-8

口

[22] H.-K. Kim, S. G. Kim, W. Dong, I. Steinbach, B.-J. Lee, Phase-field modeling for 3D grain growth based on a grain boundary energy database, Modelling and Simulation in Materials Science and Engineering 22 (3) (2014) 034004. doi: $10.1088 / 0965-0393 / 22 / 3 / 034004$ URL http://stacks.iop.org/0965-0393/22/i=3/a=034004?key= crossref.93bafc2174026807f818a4ef5b7aa405

[23] B. Merriman, J. K. Bence, S. J. Osher, Motion of Multiple Junctions: A Level Set Approach Journal of Computational Physics 112 (2) (1994) 334-363. doi:10.1006/jcph.1994.1105

765 URL http://linkinghub.elsevier.com/retrieve/pii/ S0021999184711053 
[24] M. Bernacki, Y. Chastel, T. Coupez, R. Logé, Level set framework 口. for the numerical modelling of primary recrystallization in polycrystalline materials, Scripta Materialia 58 (12) (2008) 1129-1132. doi:10.1016/j.scriptamat.2008.02.016

口 URL http://linkinghub.elsevier.com/retrieve/pii/ S1359646208001425

[25] M. Elsey, S. Esedoglu, P. Smereka, Large-scale simulation of normal grain growth via diffusion-generated motion, Proceedings of the Royal Society A: Mathematical, Physical and Engineering Sciences 467 (2126) (2011) 381-401. doi:10.1098/rspa.2010.0194

[26] M. Elsey, S. Esedoglu, P. Smereka, Diffusion generated motion for grain growth in two and three dimensions, Journal of Computational Physics 228 (21) (2009) 8015-8033. doi:10.1016/j.jcp.2009.07.020.

URL http://dx.doi.org/10.1016/j·jcp.2009.07.020

[27] C. Mießen, M. Liesenjohann, L. Barrales-Mora, L. Shvindlerman, G. Gottstein,

a An advanced level set approach to grain growth Accounting for grain boundary anisotropy and finite triple junction mobility, Acta Materialia 99 (99) (2015) 39-48. doi:10.1016/j.actamat.2015.07.040.

URL http://linkinghub.elsevier.com/retrieve/pii/ S135964541500511X

[28] R. Logé, M. Bernacki, H. Resk, L. Delannay, H. Digonnet, Y. Chastel,

घ T. Coupez, Linking plastic deformation to recrystallization in metals using digital microstructures, Philosophical Magazine 88 (30-32) (2008) 3691-3712. doi : $10.1080 / 14786430802502575$.

口 URL http://www.tandfonline.com/doi/abs/10.1080/ 14786430802502575

[29] M. Bernacki, R. Logé, T. Coupez, Level set framework for the finite1 element modelling of recrystallization and grain growth in polycrys795 talline materials, Scripta Materialia 64 (6) (2011) 525-528. doi: 
$10.1016 /$ j.scriptamat.2010.11.032

(1)

URL http://linkinghub.elsevier.com/retrieve/pii/ S1359646210007906

[30] H. Hallberg, A modified level set approach to 2D modeling of dynamic recrystallization, Modelling and Simulation in Materials Science and Engineering 21 (8) (2013) 085012. doi:10.1088/0965-0393/21/8/085012. URL http://stacks.iop.org/0965-0393/21/i=8/a=085012?key= crossref.fc2d86d9805f0075a83535b0524735cb

[31] B. Scholtes, M. Shakoor, A. Settefrati, P.-O. Bouchard, N. Bozzolo, M. Bernacki, New finite element developments for the full field modeling of microstructural evolutions using the level-set method, Computational Materials Science 109 (2015) 388-398. doi:10.1016/j . commatsci.2015.07.042. URL http://linkinghub.elsevier.com/retrieve/pii/ S0927025615004528

[32] B. Scholtes, R. Boulais-Sinou, A. Settefrati, D. Pino Muñoz, I. Poitrault, A. Montouchet, N. Bozzolo, M. Bernacki, 3D level set modeling of static recrystallization considering stored energy fields, Computational Materials Science 122 (2016) 57-71. doi:10.1016/j.commatsci.2016.04.045

प URL http://linkinghub.elsevier.com/retrieve/pii/ S0927025616302142

[33] J. Kroc, Application of Cellular Automata Simulations to Modelling of Dynamic Recrystallization, Lecture Notes in Computer Science 2329 (2002) 773-782.

[34] W. Chuan, Y. He, L. H. Wei, Modeling of discontinuous dynamic recrystal1 lization of a near- $\alpha$ titanium alloy IMI834 during isothermal hot compression 820 by combining a cellular automaton model with a crystal plasticity finite el-

a ement method Computational Materials Science 79 (2013) 944-959. doi: 10.1016/j.commatsci.2013.08.004 URL http://dx.doi.org/10.1016/j.commatsci.2013.08.004 
[35] Y. Mellbin, H. Hallberg, M. Ristinmaa, A combined crystal plasticity and

[39] H. Li, X. Sun, H. Yang, A three-dimensional cellular automata-crystal plasticgraph-based vertex model of dynamic recrystallization at large deformations, Modelling and Simulation in Materials Science and Engineering 23 (4) (2015) 045011. doi:10.1088/0965-0393/23/4/045011. URL http://www.scopus.com/inward/record.url?eid=2-s2. 0-84928999862\{\&\}partnerID=tZOtx3y1

[36] E. Popova, Y. Staraselski, A. Brahme, R. K. Mishra, K. Inal, Coupled crystal plasa ticity - Probabilistic cellular automata approach to model dynamic recrystallization in magnesium alloys, International Journal of Plasticity 66 (2015) 85-102. doi:10.1016/j.ijplas.2014.04.008 URL http://dx.doi.org/10.1016/j.ijplas.2014.04.008

[37] P. Zhao, T. Song En Low, Y. Wang, S. R. Niezgoda, An integrated full-field model of concurrent plastic deformation and microstructure evolution: Application to 3D simulation of dynamic recrystallization in polycrystalline copper. International Journal of Plasticity 80 (2016) 38-55. arXiv:1509.04953, doi:10.1016/j.ijplas.2015.12.010 URL http://dx.doi.org/10.1016/j.ijplas.2015.12.010

[38] L. Reyes, P. Páramo, A. Salas Zamarripa, M. de la Garza, M. Guer1 rero Mata, Grain size modeling of a Ni-base superalloy using cellular automata algorithm, Materials \& Design 83 (2015) 301-307. doi:10.1016/j.matdes.2015.06.068

URL http://linkinghub.elsevier.com/retrieve/pii/ S0264127515004189

ity fi nite element model for predicting the multiscale interaction among heterogeneous deformation, DRX microstructural evolution and mechanical responses in titanium alloys, International Journal of Plasticity 87 (2016) 154-180. doi:10.1016/j.ijplas.2016.09.008

URL http://dx.doi.org/10.1016/j.ijplas.2016.09.008 
[40] M.-S. Chen, W.-Q. Yuan, H.-B. Li, Z.-H. Zou, Modeling and simulation of

n. dynamic recrystallization behaviors of magnesium alloy AZ31B using cellular automaton method, Computational Materials Science 136 (2017) 163-172. doi:10.1016/j.commatsci.2017.05.009.

口 URL http://linkinghub.elsevier.com/retrieve/pii/ S0927025617302392

[41] F. Tancret, E. Galindo-Nava, P. E. J. Rivera Díaz-del-Castillo, Dynamic recrystallisation model in precipitation-hardened superalloys as a tool for the joint design of alloys and forming processes Materials \& Design 103 (2016) 293-299. doi:10.1016/j.matdes.2016.04.076 URL http://linkinghub.elsevier.com/retrieve/pii/ S0264127516305561

[42] F. Chen, Z. Cui, J. Chen, Prediction of microstructural evolution during hot forging, Manufacturing Review 1 (2014) 6. doi:10.1051/mfreview/2014006 URL http://mfr.edp-open.org/10.1051/mfreview/2014006

[43] Y. Lin, K.-K. Li, H.-B. Li, J. Chen, X.-M. Chen, D.-X. Wen, New a constitutive model for high-temperature deformation behavior of inconel 718 superalloy, Materials \& Design 74 (74) (2015) 108-118. doi:10.1016/j.matdes.2015.03.001 URL http://linkinghub.elsevier.com/retrieve/pii/ S026130691500076X

[44] M. Azarbarmas, M. Aghaie-Khafri, J. M. Cabrera, J. Calvo, Microstruc875 tural evolution and constitutive equations of Inconel 718 alloy under quasi-static and quasi-dynamic conditions, JMADE 94 (2016) 28-38. doi:10.1016/j.matdes.2015.12.157

1 URL http://ac.els-cdn.com/S0264127515310170/

1- 1-s2.0-S0264127515310170-main.pdf?\{_\}tid=

880 8a2afa72-66d4-11e7-abc7-00000aacb35f $\{\&\} a c d n a t=$

1499845049\{_\}5e4ccb87f374a6a8a8030fc2eb25a74c 
[45] D. Weaire, J. P. Kermode, J. Wejchert, On the distribution of cell areas in a Voronoi network, Philosophical Magazine Part B 53 (5) (1986) L101-L105. doi:10.1080/13642818608240647.

[46] Z. Fan, Y. Wu, X. Zhao, Y. Lu, Simulation of polycrystalline structure

1 with Voronoi diagram in Laguerre geometry based on random closed packing of spheres, Computational Materials Science 29 (3) (2004) 301-308. doi:10.1016/j.commatsci.2003.10.006. URL http://linkinghub.elsevier.com/retrieve/pii/ S0927025603002209

[47] M. Bernacki, H. Resk, T. Coupez, R. E. Logé, Finite element model of primary recrystallization in polycrystalline aggregates using a level set framework, Modelling and Simulation in Materials Science and Engineering 17 (6) (2009) 064006. doi:10.1088/0965-0393/17/6/064006

1 URL http://stacks.iop.org/0965-0393/17/i=6/a=064006?key= crossref.b10c4b90e783241fcb950f2c83e5932f

[48] R. Quey, P. Dawson, F. Barbe, Large-scale 3D random polycrystals for the finite element method: Generation, meshing and remeshing, Computer Methods in Applied Mechanics and Engineering 200 (17-20) (2011) 1729-1745. doi:10.1016/j.cma.2011.01.002. URL http://linkinghub.elsevier.com/retrieve/pii/ S004578251100003X

905 [49] K. Hitti, P. Laure, T. Coupez, L. Silva, M. Bernacki, Precise generation of coma plex statistical Representative Volume Elements (RVEs) in a finite element con1. text, Computational Materials Science 61 (2012) 224-238. doi:10.1016/j. commatsci.2012.04.011.

URL http://dx.doi.org/10.1016/j.commatsci.2012.04.011

${ }_{910}$ [50] L. Chen, J. Chen, R. A. Lebensohn, Y. Z. Ji, T. W. Heo, S. Bhattacharyya, 
K. Chang, S. Mathaudhu, Z. K. Liu, L. Q. Chen, An integrated fast Fourier transform-based phase-field and crystal plasticity approach to model recrystallization of three dimensional polycrystals, Computer Methods in Applied Mechanics and Engineering 285 (2015) 829-848. doi:10.1016/j.cma.2014.12. 007.

[51] U. F. Kocks, Laws for Work-Hardening and Low-Temperature Creep, Journal of Engineering Materials and Technology 98 (1) (1976) 76. doi:10.1115/1.3443340.

URL http://materialstechnology.asmedigitalcollection.asme. org/article.aspx?articleid=1422062

[52] H. Mecking, U. Kocks, Kinetics of flow and strain-hardening, Acta Metallurgica 29 (11) (1981) 1865-1875. doi:10.1016/0001-6160(81)90112-7.

[53] A. Yoshie, H. Morikawa, Y. Onoe, K. Itoh, Formulation of static recrystallization of austenite in hot rolling process of steel plate., Transactions of the Iron and Steel Institute of Japan 27 (6) (1987) 425-431. doi:10.2355/isijinternational1966.27.425.

口 URL http://joi.jlc.jst.go.jp/JST.Journalarchive/ isijinternational1966/27.425?from=CrossRef

[54] F. J. Humphreys, M. Hatherly, Recrystallization and related annealing phenomena, Elsevier, 2004.

[55] J. E. Bailey, P. B. Hirsch, The Recrystallization Process in Some Polycrystalline Metals, Proceedings of the Royal Society A: Mathematical, Physical and Engineering Sciences 267 (1328) (1962) 11-30. doi:10.1098/rspa.1962.0080 URL http://rspa.royalsocietypublishing.org/cgi/doi/10.1098/ rspa.1962.0080

[56] P. Peczak, M. J. Luton, The effect of nucleation models on dynamic recrystallization I. Homogeneous stored energy distribution. Philosophical Magazine Part B 68 (1) (1993) 115-144. doi:10.1080/13642819308215285. 
URL http://www.tandfonline.com/doi/abs/10.1080/

940 13642819308215285

[57] K. Huang, Towards the modelling of recrystallization phenomena in multi-pass conditions: application to 304L steel, Ph.D. thesis (2012).

URL https: //pastel .archives-ouvertes.fr/pastel-00682138

[58] M. Shakoor, B. Scholtes, P.-O. Bouchard, M. Bernacki, An efficient and parallel

level set reinitialization method Application to micromechanics and microstructural evolutions, Applied Mathematical Modelling 39 (23-24) (2015) 7291-7302. doi:10.1016/j.apm.2015.03.014. URL http://linkinghub.elsevier.com/retrieve/pii/ S0307904X15001638 